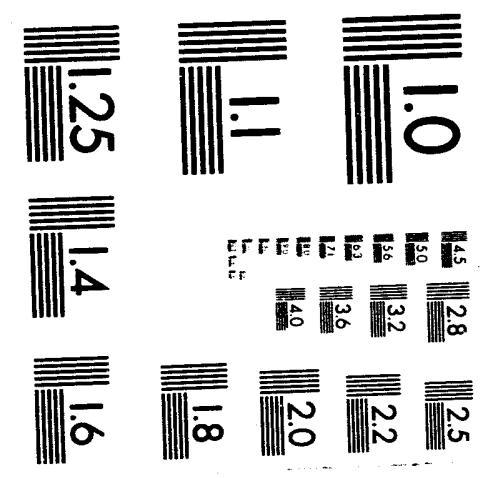



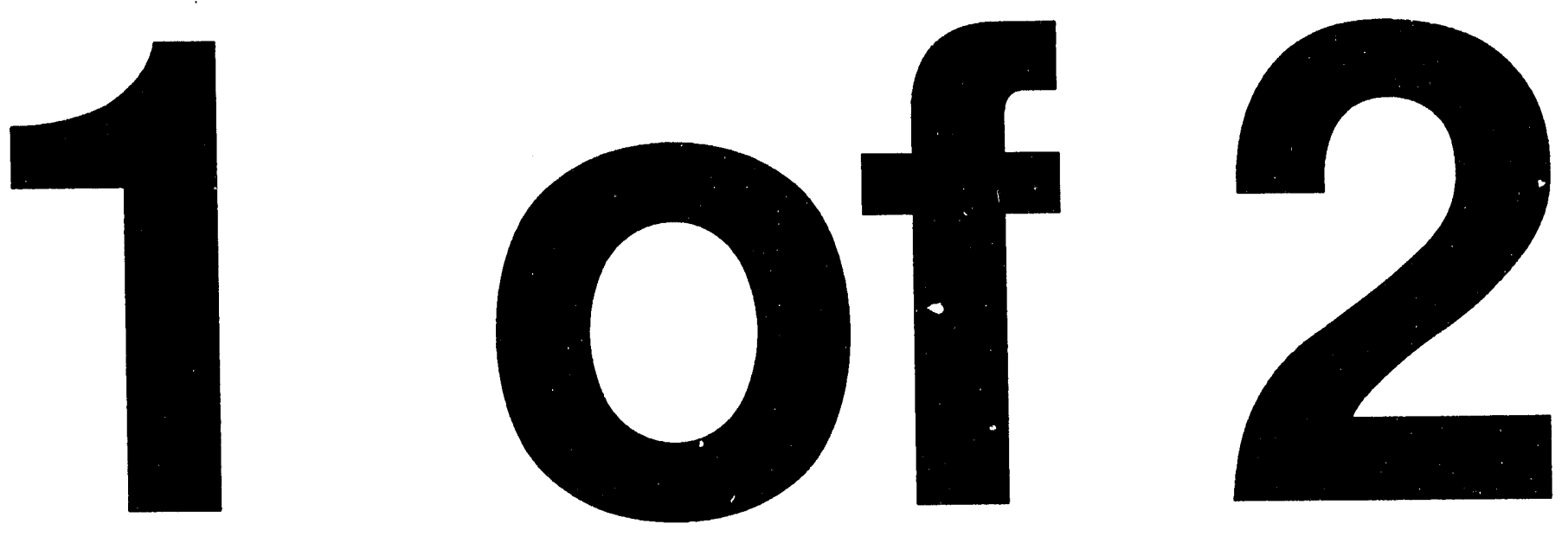


\title{
An Axial Approach To Detection in Capillary Electrophoresis
}

\author{
by John Aaron Taylor
}

thesis submitted to Iowa State University

Ames Laboratory, U. S. DOE

Iowa State University

Ames, Iowa 50011

Date Transmitted: May 1993

\author{
Prepared for the U. S. Department of Energy \\ Under contract no. W-7405-eng-82.
}

\section{DISCLAIMER}

This report was prepared as an account of work sponsored by an agency of the United States Government. Neither the United States Government nor any agency thereof, nor any of their employees, makes any warranty, express or implied, or assumes any legal liability or responsibility for the accuracy, completeness, or usefulness of any information, apparatus, product, or process disclosed, or represents that its use would not infringe privately owned rights. Reference herein to any specific commercial product, process, or service by trade name, trademark, manufacturer, or otherwise does not necessarily constitute or imply its endorsement, recommendation, or favoring by the United States Government or any agency thereof. The views and opinions of authors expressed herein do not necessarily state or reflect those of the United States Government or any agency thereof.

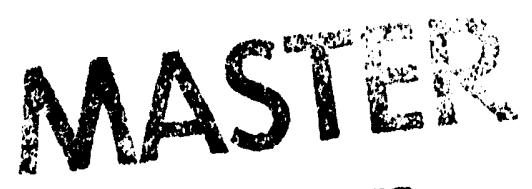


An axial approach to detection

in capillary electrophoresis

John Aaron Taylor

Under the supervision of Edward $s$. Yeung

From the Department of Chemistry

Iowa State University

The purpose of this research has been to develop and demonstrate visualization schemes which further the capabilities of capillary electrophoresis instrumentation. Our approach involves on-axis illumination of the compounds inside the capillary detection region and is applied to absorbance and fluorescence detection.

Absorbance measurements were accomplished by focussing an incident beam of laser light into one end of the separation capillary. By utilizing signals collected over the entire length of the analyte band, this technique enhances the analytical path length of conventional absorbance detection sixty fold. The demonstrated instrument offers a fifteen-fold improvement in concentration limits of detection.

Three fluorescence detection experiments are discussed; all of which involve the insertion of a small optical fiber into the capillary to introduce the excitation beam from a laser source. The first of these 
employs a high refractive index liquid phase to satisfy total internal reflectance along the capillary axis, thus greatly reducing light scatter from capillary walls. The second utilizes a charge-coupled device (CCD) camera for simultaneous imaging of an array of capillaries (a technique that may prove useful in high information throughput tasks such as genome sequencing). The third is basic study of fluid motion inside the capillary under conditions of pressure-driven and electroosmotic flow. In this study, the CCD is used to track nanometer-sized particles as well as fluorescent bands to give a quantitative, empirical assessment of the factors influencing capillary electrophoresis separations. 
An axial approach to detection

in capillary electrophoresis

by

John Aaron Taylor

A Dissertation Submitted to the

Graduate Faculty in Partial Fulfillment of the

Requirements for the Degree of

DOCTOR OF PHILOSOPHY

Department: Chemistry

Major: Analytical Chemistry

Approved:
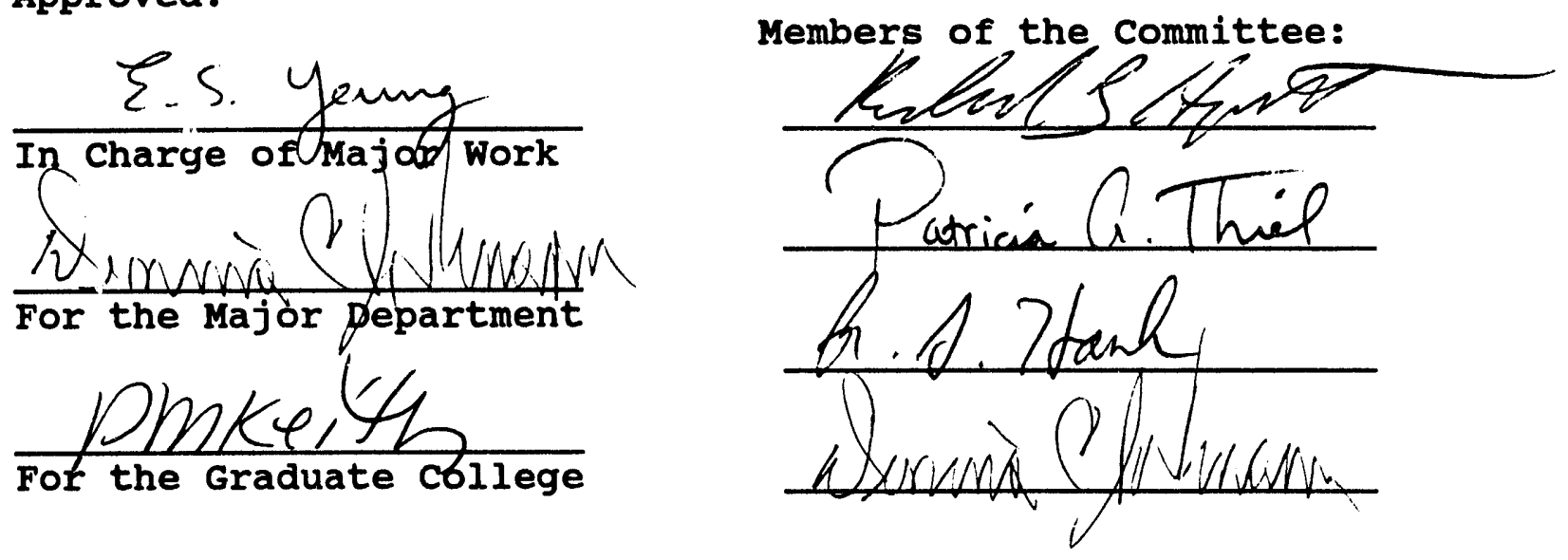

Iowa state University

Ames, Iowa 
TABLE OF CONTENTS

ACKNOWLEDGEMENTS $\ldots \ldots \ldots \ldots \ldots \ldots \ldots \ldots \ldots \ldots \ldots \ldots \ldots \ldots \ldots$ iv

GENERAL INTRODUCTION $\ldots \ldots \ldots \ldots \ldots \ldots \ldots \ldots \ldots \ldots \ldots \ldots \ldots$

LITERATURE REVIEW ......................... 3

PAPER 1. AXIAL-BEAM ABSORBANCE DETECTION FOR CAPILLARY ELECTROPHORESIS ......... 13

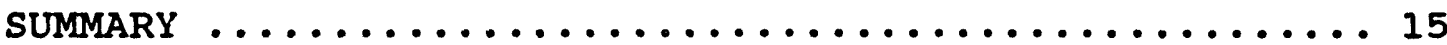

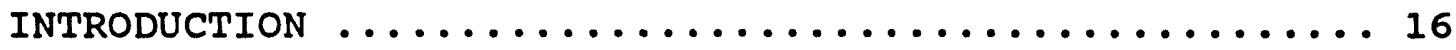

EXPERIMENTAL ......................... 17

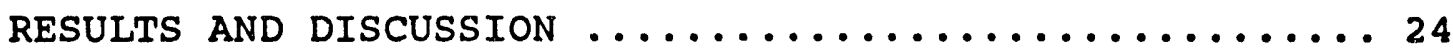

ACKNOWLEDGEMENTS $\ldots \ldots \ldots \ldots \ldots \ldots \ldots \ldots \ldots \ldots \ldots \ldots \ldots \ldots$

REFERENCES $\ldots \ldots \ldots \ldots \ldots \ldots \ldots \ldots \ldots \ldots \ldots \ldots \ldots \ldots \ldots \ldots \ldots$

PAPER 2. AXIAL-BEAM LASER-EXCITED FLUORESCENCE DETECTION IN CAPILLARY ELECTROPHORESIS • 40

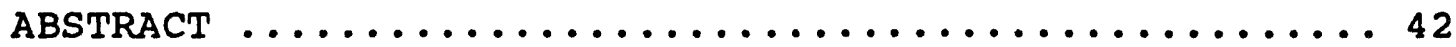

INTRODUCTION $\ldots \ldots \ldots \ldots \ldots \ldots \ldots \ldots \ldots \ldots \ldots \ldots \ldots \ldots$

EXPERIMENTAL SECTION .....................46

RESULTS AND DISCUSSION ........................ 51

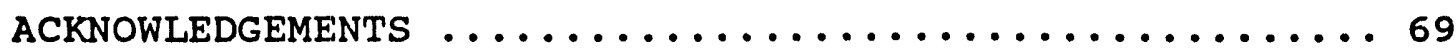

LITERATURE CITED ...................... 70 
PAPER 3. MULTIPLEXED FLUORESCENCE DETECTOR FOR CAPILLARY ELECTROPHORESIS USING AXIAL OPTICAL FIBER ILLUMINATION $\ldots \ldots \ldots \ldots \ldots 71$

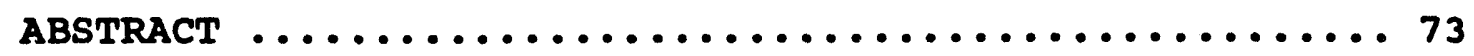

INTRODUCTION $\ldots \ldots \ldots \ldots \ldots \ldots \ldots \ldots \ldots \ldots \ldots \ldots \ldots \ldots$

EXPERIMENTAL SECTION $\ldots \ldots \ldots \ldots \ldots \ldots \ldots \ldots \ldots \ldots \ldots$

RESULTS $\ldots \ldots \ldots \ldots \ldots \ldots \ldots \ldots \ldots \ldots \ldots \ldots \ldots \ldots \ldots$

DISCUSSION $\ldots \ldots \ldots \ldots \ldots \ldots \ldots \ldots \ldots \ldots \ldots \ldots \ldots . \ldots 9$

ACKNOWLEDGEMENTS $\ldots \ldots \ldots \ldots \ldots \ldots \ldots \ldots \ldots \ldots \ldots \ldots . \ldots 9$

REFERENCES $\ldots \ldots \ldots \ldots \ldots \ldots \ldots \ldots \ldots \ldots \ldots \ldots \ldots \ldots$

PAPER 4. INVESTIGATION OF ELECTROKINETIC FLOW

USING CHARGE-COUPLED DEVICE IMAGING ....102

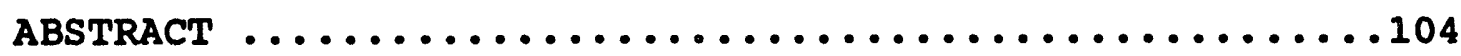

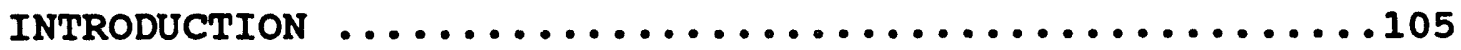

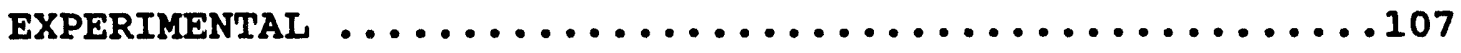

RESULTS AND DISCUSSION $\ldots \ldots \ldots \ldots \ldots \ldots \ldots \ldots \ldots \ldots \ldots \ldots$

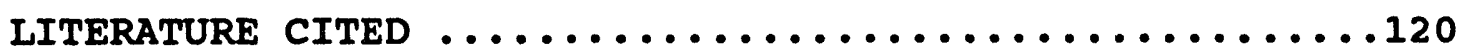

GENERAL CONCLUSIONS $\ldots \ldots \ldots \ldots \ldots \ldots \ldots \ldots \ldots \ldots \ldots \ldots \ldots$

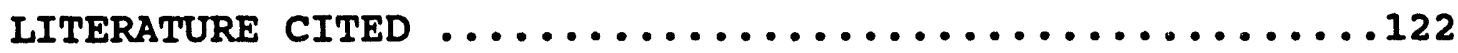




\section{ACKNONLEDGEYENTS}

This work was performed at Ames Laboratory under contract no. W-7405-eng-82 with the U.S. Department of Energy. The United States government has assigned the DOE Report number IS-T 1656 to this dissertation.

I am indebted beyond repayment to so many people.

I thank Dr. Yeung for acquiring funding for this research, for my education as a researcher, and for his patience over the last six years. My graduate committee, Drs. Honzatko, Houk, Johnson, and Thiel, have honored me by investing time and showing interest in my development as a scientist. I am especially grateful to Dr. Houk and Dr. Johnson. Without their encouragement in my first year at Iowa state, I probably wouldn't be writing this.

My coworkers in the Yeung group have been a constant source of companionship and entertainment. An additional thanks to Tommy Wayne Garner and Lance "Is this our bus?" Koutny, who stood up with me when I said my marriage vows. I haven't forgotten Tracy Koutny and Art Grunke who added a bag of Cheetos and some well-applied shoe polish to that important weekend. I also want to thank all of the new friends who helped me celebrate my 28 th birthday and Dave 
McGregor and Sheri Lillard for keeping a roof over our heads during the 1992 tiger invasion.

Back in my home state, Illinois (said with a slow, longing breath), I have an army of supporters called aunts and uncles. These people have done everything from sending gifts and cards to hauling "tons" of furniture 350 miles to furnish my apartment. Some of their names are Bertha and William Glore, Aileen and Bernie Moore, Gladys Tilton, and Dora and John Ritchie. With this group, I also thank my cousin Tricia Cox, now a graduate student at North Carolina state, for all the letters and phone calls, and for letting me watch someone else pursue graduate education after a chemistry degree from Millikin.

All of my life, my parents, Jack and Freda Taylor, have been a source of wisdom and love. My hope is that someday people will know how much these two have given to me by what I can give to others.

Then there is my wife of almost four years, Karalee. Before I met this woman, I felt content with seeing black, white, and a few shades of grey. Now, I cannot wait to behold the shades and combinations of red, yellow, and blue that she has in store for me.

Above all else, I thank the I Am who, out of His love, takes part in our learning about who He is and what He has made. 


\title{
GENERAL INTRODUCTION
}

\begin{abstract}
"Opposites attract." This two-word sentence and principle has become invaluable in describing the mechanics of magnetism, interpersonal relationships, Velcro, music, and, more relevant to this dissertation, the movement of ions in an electrical field. The most elaborate research is built with simple principles. Before I started this research, capillary electrophoresis (CE), the separation of mixtures of ions along the length of a thin capillary under a large electric field gradient, had already matured into a widely acknowledged and useful technique. The challenge was to develop ways of detecting compounds of interest that specifically fit the new demands of $C E$ separations; oncolumn detection, low mass detection limits, and high information throughput. All of this has led to a new generation of detectors for flowing systems, many based on well-known principles of spectroscopy and electrochemistry.
\end{abstract}

An Explanation of the Dissertation Organization This general introduction and a literature review precede three research papers which have been published in major analytical chemistry journals and a fourth that will 
soon be submitted for publication. The papers are followed by a general conclusions section. A literature-cited section follows the general conclusions and contains references for the general introduction and general conclusions sections. References for each of the four papers follow the paper in which they are cited. 


\section{LITERATURE REVIEW}

This literature review is comprised of three sections. The first two explain, in detail, two of the most common detection modes used with capillary electrophoresis and introduce the problems addressed in the first two papers. The last section introduces the challenge of the human genome initiative and the role CE may play therein. Absorbance detection in capillary electrophoresis

The nearly universal applicability of ultraviolet and visible absorbance detectors has made them commonplace in commercial capillary electrophoresis instruments. Practically every compound of interest (aromatics, organic acids, proteins, DNA fragments, etc.) exhibits some analytically useful absorbance between $200 \mathrm{~nm}$ and $250 \mathrm{~nm}$. This technique may have been an easy choice for separation scientists specializing in high performance liquid chromatography who used absorbance detection at $254 \mathrm{~nm}$ all through the seventies. The smaller internal diameters of capillary electrophoresis columns have also reduced liquid phase absorption to allow detection at lower wavelengths (220 nm range), thus allowing the use of non-aromatic transitions for organic compounds. 
Several designs have been applied to absorbance detection, all of which contain elements for light introduction from a source, through the capillary, and onto a photon detector for quantitation. Typical light sources include cd "panray"l, mercury", and deuterium lamps. The use of lasers for absorbance detection may prove advantageous because of their high intensity and relative ease of focus, but has nct become commonplace due to intensity fluctuations and monetary considerations.

Detector designs do, however, vary greatly in the way that light is introduced into the capillary. Perhaps the most simple design was introduced by Jorgenson and coworkers $^{1}$. In that case, a $100 \mu \mathrm{m}$ pinhole was sandwiched between a "pen-ray" light source and the capillary with an interference filter and photomultiplier tube on the other side of the capillary for signal collection. A common scheme used in commercial instruments is to focus light from a source using a ball lens mounted near the capillary wall. This provides a rugged, cost effective, easy-to-use detector. When the capillary electrophoresis instrumentation is to be interfaced with existing equipment, two optical fibers can be placed on opposing walls, one for introduction and one for collection. 2 
The aspiration of absorbance detection research is to obtain the lowest detection limits. Assuming that all of the incident monochromatic beam follows one path through the detection region, the absorbance measured follows Beer's Law:

$$
A=\epsilon b c
$$

where: $\quad \epsilon=$ molar absorptivity of the analyte

$b=$ analytical pathlength

$c=$ molar concentration

One useful way to compare limits of detection that is independent of molar absorptivity is to calculate the ratio ALOD / b

where:

$$
\text { ALOD }=\log \frac{\text { Incident Beam Intensity }}{\text { Incident Intensity }-(3 \times \text { Noise })}
$$

Even though the approximations made here oversimplify the optical constraints on light introduction into a small cylindrical capillary, the second ratio does suggest two directions to lower detection limits (decreasing ALOD or increasing b).

Decreasing the absorbance limit of detection (ALOD) requires minimizing the noise in the incident light beam. While reference beams can be used to compensate for flicker noise in the light source, until now the electronic 
circuitry for true cancellation has been lacking. The Yeung group 16 has recently utilized a new approach to electronic subtraction of the analytical signal from a reference. The device described is based on a circuit first applied to optical systems by Hobbs 17 and is capable of a noise-to-signal ratio of $3 \times 10^{-7}$ for the subtracted incident light source.

An alternative direction is to increase the number of analyte molecules involved in absorbing the incident light beam by exposing a length of the capillary through a slit set lengthwise, parallel to the capillary axis. This would have a similar effect to increasing the analytical pathlength, b. Wang, Hartwick, and Champlin ${ }^{3}$ designed an adjustable aperture-width detector cell to demonstrate the utility of this approach. The problem in this case is that the slit length must be "fine-tuned" for each particular application to avoid band broadening.

The only way to truly increase $b$ to the full length of the analyte band without contributing to peak width is to introduce and collect light in line with the capillary axis. Absorption detection utilizing this axial-beam method was first demonstrated by $X i$ and $Y{ }^{4}{ }^{4}$ for capillary liquid chromatography. A laser beam was focussed into one end of the separation capillary and propagated 
along the capillary axis by total internal reflectance. The light that traversed the entire capillary length was collected with a lens and imaged onto a photomultiplier tube. The application of on-axis, laser-based absorbance detection to capillary electrophoresis is the topic of the first paper of this dissertation. Fluorescence detection in capillary electrophoresis

In contrast to absorbance detection, the sensitivity of a fluorescence detector does not necessarily depend on the analytical pathlength ${ }^{5}$ or light source stability. Sensitivity depends on the intensity of the incident light beam and extinction coefficient and quantum efficiency of the fluorophor. Since single photons of light can be measured without difficulty, the sensitivity of photon transducers is not a limitation. Detection limits, which are inversely proportional to the signal-to-noise ratio at the lowest measured signal, should then depend on the discrimination between the fluorescence signal and the background signal produced by scatter from cell walls, solvent, or impurities.

one approach to minimizing this background was introduced by Dovichi et al. who used a sheath flow cell adapted from a flow cytometer ${ }^{6}$. The sheath flow which surrounded the sample stream was contained in a square 
cell. Another simple but effective way to discriminate against scatter has been patented by the Yeung group. The key to this technique is the formation of a very sharply focussed image of the capillary centered on a spatial filter which transmits only the fluorescence signal emanating from the center of the capillary. This detection scheme has been used to detect the contents of a single red blood cell. 18,19

While the above offer excellent detection limits, alignment and preparation of the capillary beforehand could prohibit their use in some applications. Removal of protective coatings, which usually contribute greatly to background fluorescence, is usually accomplished by placing the capillary in a flame, or for a milder treatment, boiling sulfuric acid. In the second paper of this dissertation, a detector is discussed which requires neither difficult alignment nor preparation of the outside capillary surface.

The human genome initiative

In 1988, the United States Congress allocated funds to the National Institutes of Health (NIH) and the Department of Energy (DOE) to "determine the structure of complex genomes."7 The selling points of this initiative were numerous. A completely mapped and sequenced genetic code 
could provide invaluable insight into understanding more than 400 known genetic disorders as well as many other diseases where certain genetic sequences may contribute. Diagnosis of genetic disorders before or shortly after birth would afford an opportunity to avoid circumstances which contribute to the disease. 8 The code would also help scientists unravel mysteries such as cell differentiation and development. This mapping and sequencing would not only include the human genome, but also more "experimentally accessible" organisms such as E. coli bacterium, Saccharomyces cerevisiae yeast, the nematode worm, the fruit fly, mice, and a selected plant. These studies may point out key protein-encoding sequences related to analogous biological processes in humans. 10 Directed research began in 1990 and is expected to take fifteen years to reach completion.

The human genome consists of 50,000 to 100,000 genes on 23 pairs of chromosomes. With two thousand to two million base pairs per chromosome, it is estimated that three billion base pairs will eventually need to be sequenced. The regions responsible for encoding protein sequences (exons), however, make up only $5 \%$ of the code. 9 To distinguish exons from non-coding regions (introns), the 
study of messenger mRNA, which contains only exons, may also be necessary.

since current sequencing technology is only capable of determining about five hundred base pairs per run, the way that the human genome is cut into smaller pieces will be crucial to the success of the project. Mapping, determining the locations of genes on chromosomes, is the key to dividing the genome for sequencing in a way that can be reconstructed and that will yield the largest amount of useful information in the shortest period of time. Genetic mapping is the study of the way certain genetic sequences are linked, or passed along together from generation to generation. Physical mapping describes the location of each gene in terms of base pairs and visible landmarks. Both disciplines will be necessary in completion and application of sequence data.

This initiative will bring about the development of technologically advanced tools and techniques which will be needed to carry out the tasks described. Pulsed-field gel electrophoresis, polymerase chain reaction, and restriction fragment linked polymorphisms are just a few of the modern technologies which are being applied to mapping large sections of DNA. 7 Frederick Sanger is given most of the credit for developing the first DNA sequencing method in 
the early 1970's.11 The Sanger method produces small complimentary fragments of radiolabeled DNA each terminated with one of four dideoxynucleotides (ddATP, ddCTP, ddTTP, and ddGTP). Another sequencing method was developed by Gilbert and Maxam a few years later. In this case, instead of complimentary segments with radiolabeled primers, a single radiolabled strand is separated and chemically cut at specific nucleotides using different reartion mixtures. Whether the Sanger or Maxam-Gilbert method is used, a set of short DNA fragments from each of four reaction mixtures must be separated by length to read the sequence. To reduce the number of separations needed by a factor of four, two groups 12,13 have introduced a system that tags each of the four terminal bases with a different fluorescent marker. The factor that is common to each of these techniques is the medium for separation of the fragments. Traditionally this tool has been gel electrophoresis. New technology in capillary electrophoresis, however, has greatly increased the speed with which these separations can be obtained.14,15,20 The reason for this is that the more efficient heat dissipation of the capillary allows application of much larger electrical fields. Since the capillary separations require minutes instead of the hours needed with slab gels, the 
next logical step toward speeding up sequencing operations is to run capillary separations in parallel. Paper three of this dissertation introduces a capillary electrophoresis detection system that is capable of simultaneously monitoring separations in multiple capillaries. 
13

PAPER 1. AXIAL-BEAM ABSORBANCE DETECTION FOR CAPILLARY ELECTROPHORESIS 


\section{AXIAL-BEAM ABSORBANCE DETECTION FOR CAPILLARY ELECTROPHORESIS}

John A. Taylor and Edward S. Yeung*

Ames Laboratory-USDOE and Department of Chemistry Iowa State University, Ames, IA 50011

Reprinted with perimission from the Journal of

Chromatography 1991, 550, 831-837. Copyright 1991 Elsevier Science Publishers B. V. 


\section{SUMMARY}

A novel approach to absorbance detection has been demonstrated for capillary electrophoresis (CE) utilizing an incident light beam directed along the capillary axis. The resulting absorption pathlength is roughly equal to the width of the sample bands present. This represents a 60-fold improvement in analytical pathlength over conventional on-column absorbance detectors without any loss of separation efficiency. The method can be used with completely aqueous buffer systems or those containing organic modifiers, and is applicable to both fused silica and teflon (PTFE) capillaries. 


\section{INTRODOCTION}

The flood of capillary electrophoresis (CE)

applications in recent years has driven the development of many detection schemes, each with its own degree of selectivity and sensitivity to fit a specific purpose. Two characteristics have allowed absorbance detection to earn its place among the most popular of these techniques. First of all, if the proper incident wavelengths are chosen (e.g., the ultraviolet region), this one detector can be used for almost every class of organic compounds. Second, this technique usually does not require sample derivatization or special buffer considerations.

Ultra high separation efficiencies and low mass detection limits, which have become the selling points of $C E$, require that detector volumes be small, thus favoring on-column detectors. By convention, on-column absorbance detection in CE has been carried out with the incident light beam directed perpendicular to the capillary walls.1-3 While some of these designs have demonstrated reasonable detection limits owing to high light source stability, they are seriously hindered by short absorption pathlengths. Diffusion processes along the axis of a CE column keep the minimum bandwidth attainable to about 


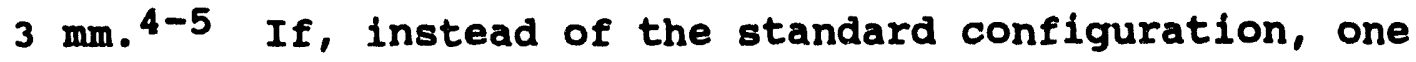
directs the light beam along the capillary, then the entire 3-mm bandwidth becomes the absorption pathlength. In a

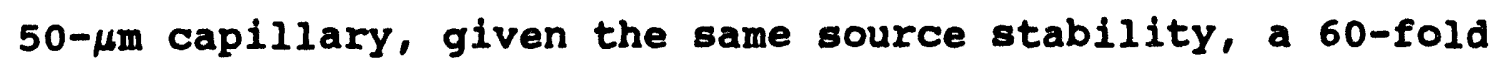
improvement in detection limits could be obtained with no loss of separation efficiency.

Increasing analytical pathlengths by directing the incident light beam along the axis of a capillary cell is not a completely new idea. Fuwa et al. have obtained analytical pathlengths in excess of one meter in glass and polymer capillaries. 6-8 Axial absorbance detection has also been demonstrated for smaller capillaries incorporating open tubular liquid chromatography. 9 In that application, a laser beam was focused into one end of a 10- $\mu \mathrm{m}$ fused silica capillary using a single-element lens. with the proper choice of solvents (i.e., solvents having a greater refractive index than that of fused silica), light entering the capillary is transmitted by total internal reflection at the interface between the solution and the capillary wall. At the capillary exit, light is collected with a microscope objective and imaged onto either a photomultiplier tube or a photodiode.

This research involves the application of the axial-beam technique to detection in CE. Two strategies 
have been studied. The first of these takes advantage of the partial internal reflectance at the water/fused silica interface to propagate light along the capillary axis. The second utilizes low refractive index polymers and buffer systems with organic modifiers to bring about tutal internal reflectance. 


\section{EXPERIMENTAI}

Axial detection system. Figure 1 is a block diagram for axial detection. The light source was a 10-mw, 632-nm HeNe laser (NEC, Mountain Valley, CA, GLG5261). The beam was directed through two mirrors on pivoting mounts (Newport, Fountain Valley, $\mathrm{CA}, 2^{n} \times 2^{n}$ ) and focused using a $2.5 \mathrm{~cm}$ focal-length lens (Newport, KPX076) mounted on a 3-dimensional translational stage. Both the front and the rear cells were constructed from plexiglass blocks with channels bored in a three dimensional $\mathrm{T}$-shape to allow introduction of the capillary, incident light beam, electrode wire, and liquid buffer. These cells contained windows fabricated from microscope slides and were sealed on all sides except the top with epoxy (Devcon, 5-minute). The capillary was mounted in a glass support (borosilicate glass tubing $5 \mathrm{~mm} 0 . \mathrm{d} ., 1 \mathrm{~mm}$ i.d., $25 \mathrm{~cm}$ in length) by gluing at both ends and through three evenly spaced holes drilled perpendicular to the length of the tube. This support was used to maintain rigidity and straightness.

Light emitted from the capillary exit was collected with a I0x microscope objective (Bausch and Lomb, Rochester, NY, BM2888, $16 \mathrm{~mm}$ ) and focused onto a spatial filter (size ranging from $1 \mathrm{~mm}$ to $10 \mathrm{~mm}$ depending on the 


\section{Figure 1.}

Schematic for axial absorbance detection in CE. The dashed lines indicate the path of the laser beam. 


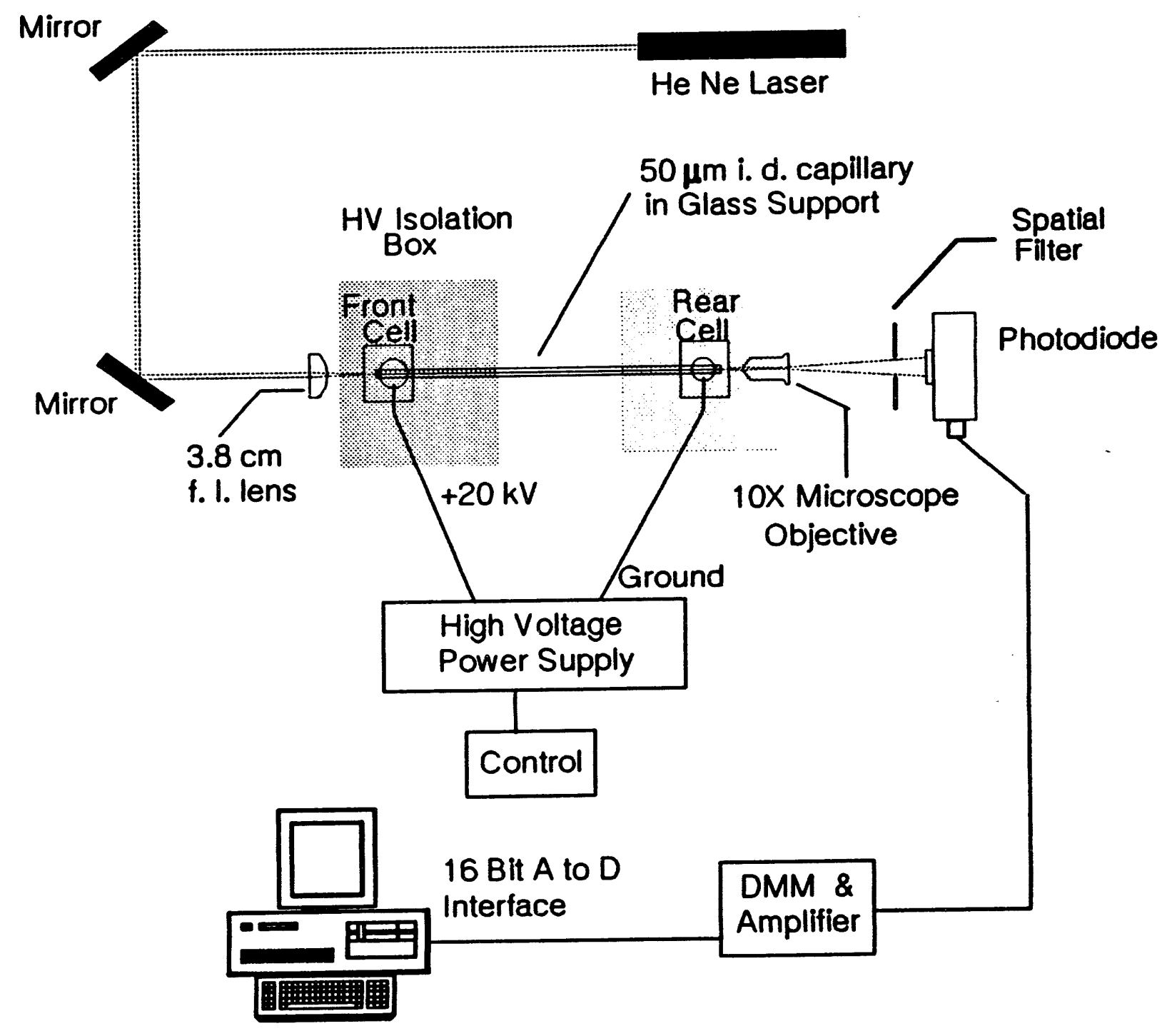


distance between the objective and the filter). The resulting beam intensity was converted to an electrical signal via a photomultiplier tube (Hamamatsu, Middlesex, NJ, R928) or a photodiode (Hamamatsu, $1 \mathrm{~cm}^{2}$ area, S179004N) and amplified through a digital multimeter (Keithley Instruments, Cleveland, $\mathrm{OH}, 160 \mathrm{~B})$. Data collection and analysis were accomplished using an IBM AT computer with a 16-bit analog-to-digital conversion board (Data Translation, Marlboro, MA, 2827). A smoothing algorithm 10 was applied to sequential data files providing an effective time constant compatible with the injection peak widths. Beam profile measurements. Intensity profiles at the exit of the capillary were measured by focusing the exiting light through the $10 \mathrm{x}$ microscope objective onto a white background at the location of the spatial aperture. Images were then recorded using a CCD camera (Photometrics, Tucson, AZ, Series 200) through a $90 \mathrm{~mm}$ macro lens (Tamron, Tokyo, Japan) and converted to 50 × 50 matrices of pixel intensities.

Electrophoresis. The separation of bromocresol green and bromothymol blue was carried out in fused silica capillary (Polymicro Technologies, Phoenix, AZ, $75 \mu \mathrm{m}$ i.d., $150 \mu \mathrm{m}$ o.d.) and in teflon capillary (zeus Industrial Products, Raritan, NJ, $50 \mu \mathrm{m}$ i.d., $150 \mu \mathrm{m}$ o.d.). The power 
supply (Glassman, Whitehouse station, NJ, PS/MJ30P0400-11) was operated at $+20 \mathrm{kV}$ applied across two chromel electrodes positioned at the ends of the capillary (capillary exit at ground). Electrokinetic injection was accomplished by placing a sample solution in the front cell, activating the power supply momentarily, then flushing the front cell with running buffer prior to the separation.

Buffers and reagents. For electrophoresis in silica capillary, the running buffer consisted of $10 \mathrm{mM}$ disodium phosphate (Fisher, Fair Lawn, NJ, certified ACS grade), adjusted to $\mathrm{pH} 7.5$ using phosphoric acid. When teflon capillary was used the buffer was $10 \mathrm{mM}$ disodium phosphate and $50 \mathrm{mM}$ sodium dodecylsulphate (Sigma, st. Louis, MO) in 50 volume-percent ethylene glycol (Fisher, purified grade), adjusted to $\mathrm{pH}$ 7.6. All buffers were degassed by applying vacuum while the buffer bottle was agitated in an ultrasonic bath. Bromocresol green and bromothymol blue were obtained from J. T. Baker (Phillipsburg, NJ) . 


\section{RE8OLT8 NWD DI8CO8BION}

Partial internal reflectance. An advantage of this mode of axial absorbance detection for CE is that it can be carried out using the same aqueous buffer solutions and fused silica capillary as in standard absorbance detection. According to Fuwa et $a 1,, 7$ the total intensity of light transmitted through a long glass capillary cell containing water is comprised of light rays taking many paths through the capillary center or through the walls. The secret of obtaining a useful analytical signal from the light emitted at the exit of a very small (less than $100 \mu \mathrm{m}$ i.d.) capillary is to separate wall-propagated light from center-propagated light. This was accomplished by imaging the exiting light beam on a spatial filter using a microscope objective. An important difference between the present work and the published results for liquid chromatographic detection ${ }^{9}$ is evident. In the latter case, much smaller diameter capillaries (approaching $5 \mu \mathrm{m}$ ) are needed to provide highly efficient separations. The smaller tubes are not suitable for transmitting light by partial internal reflectance because of the ratio of the cross-sectional areas of the liquid versus the walls is too small to allow proper discrimination. On the other hand, 
CE works quite well in capillaries up to $75-\mu \mathrm{m}$, and partial internal reflectance can be implemented.

An intensity profile at the exit end of the capillary, as seen at the spatial filter, is shown in Figure 2. The peak of intensity at the middle of the image represents light propagated through the capillary center while the ring of intensity around the outside represents wall-propagated light. Interference modes within the small bore of the tube are most likely responsible for the nodes seen in the center peak. This mode structure changes with heat buildup along the capillary or fluctuations in refractive index of the buffer. Evidence for the complete spatial separation of wall- and center-propagated light can be seen when the capillary is filled with an opaque dye solution. In this case, only the outer ring of intensity remained in the image. An aperture size for the spatial filter was chosen to allow only the center peak to pass on to the photomultiplier or photodiode, thus excluding wall-propagated light from the signal.

Figure 3 is a sample electropherogram incorporating partial internal reflectance axial absorption detection. on injection of the sample, the transmitted intensity drops to a low level due to the combined absorption of both sample components. This level is maintained as the samples 
Figure 2. Intensity profile at the exit end of a fused silica capillary. Capillary i.d. was $75 \mu \mathrm{m}, 0 . d .150 \mu \mathrm{m}$. 


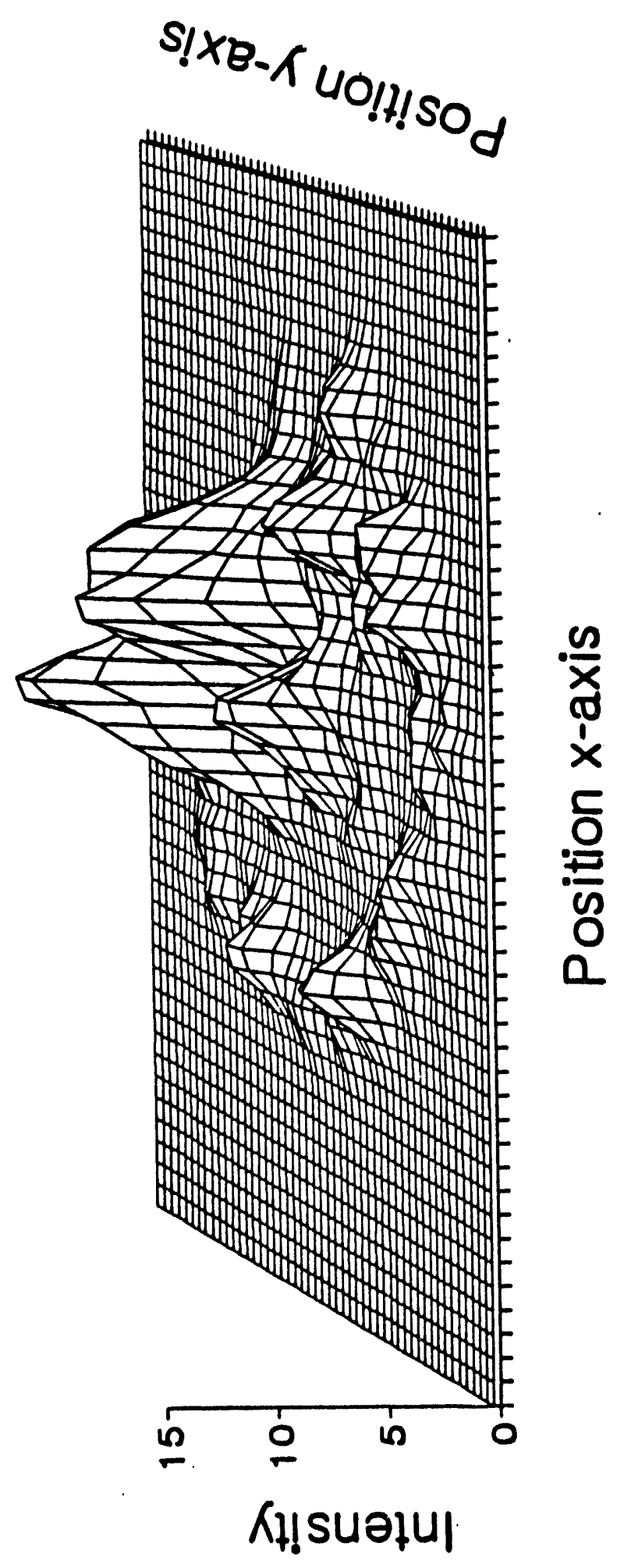


Figure 3. Separation of bromothymol blue and bromocresol green. The separation took place in a fused silica capillary (75 $\mathrm{mm}$ i.d.). Buffer: $10 \mathrm{mH}$ phosphate adjusted to $\mathrm{pH}$ 7.5. Analyte concentrations: $2 \times 10^{-5} \mathrm{H}$ each. Applied potential was $+20 \mathrm{kV}$ and electrokinetic injection time was $2.5 \mathrm{~s}$. A data smoothing routine has been applied with an effective time constant of $2.5 \mathrm{~s}$. 


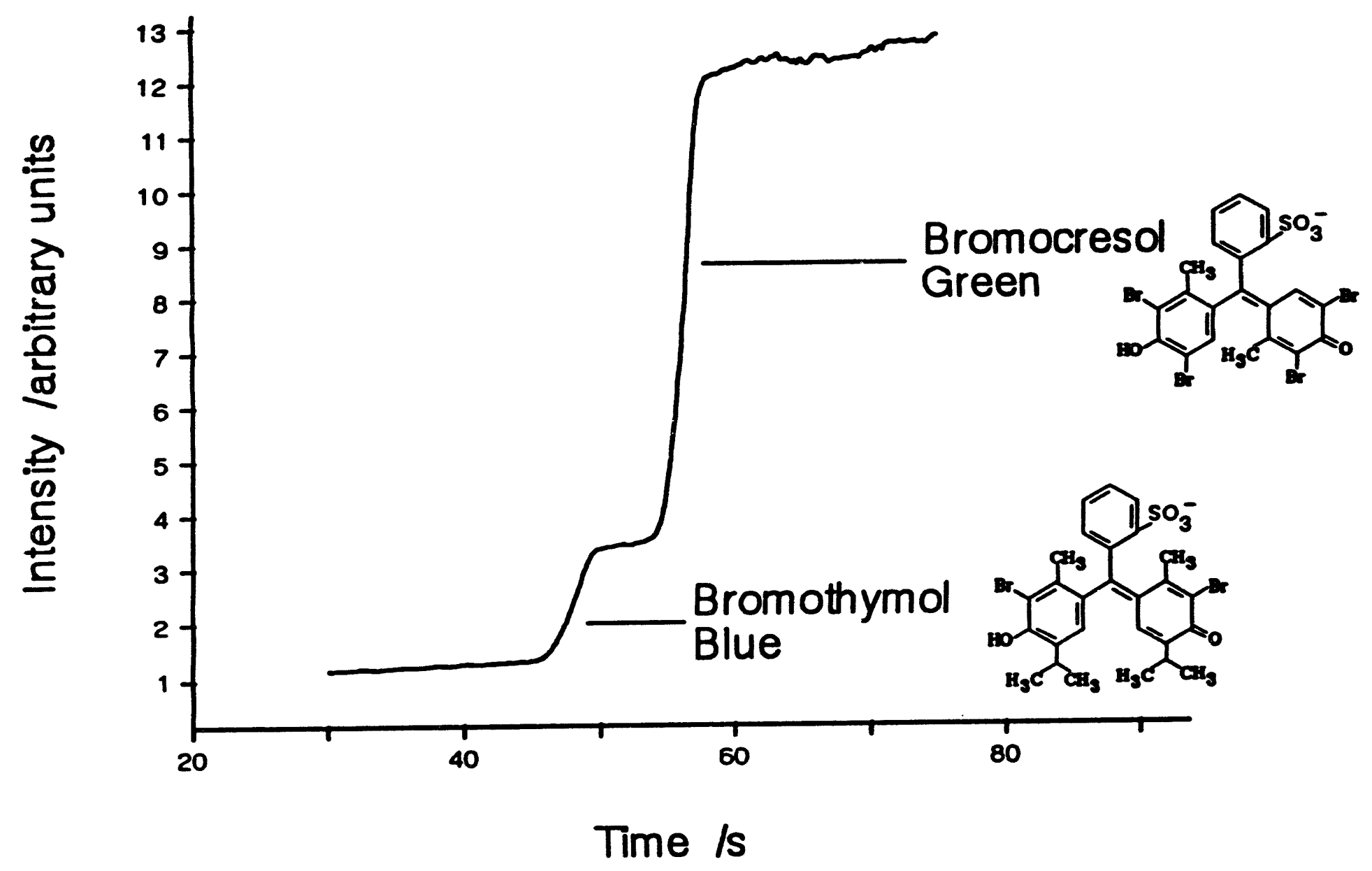


migrate within the column, even though they are physically being separated by electrophoresis. The separation of bromocresol green and bromothymol blue is indicated by two sharp rises in intensity, each representing a loss in absorbance due to the elution of the individual compounds. Bromothymol blue elutes first, owing to its larger $\mathrm{pk}_{\mathrm{a}}$ and consequently lower degree of ionization and lower affinity for the positive electrode. The "integrating" nature of this electropherogram results in lower detection limits because the absorbance of the entire length of each analyte band is measured continuously from the beginning of the run until elution.11 As has been shown previously, this type of integral electropherogram (chromatogram) preserves all the information in a normal electropherogram, such as elution time, peak area and peak width. In fact, the first derivative plot of this data produces the normal electropherogram. 9

Total internal reflectance. Total internal reflectance allows efficient transmission of light along the liquid-filled core of the capillary, analogous to an optical fiber. When this phenomenon is applied to axial detection, two distinct advantages are seen. The first advantage arises from the fact that all the light rays impinging on the capillary, within a certain cone of 
acceptance, are completely internally reflected. The results are less noise associated with small capillary vibrations in the electric field and greater reproducibility of measured absorbances. A second advantage is that since very little loss of light occurs, longer capillaries (in excess of one meter) in conjunction with higher applied potentials can be used for greater electrophoretic resolution.

The condition for total internal reflectance is that the refractive index of the liquid filling the capillary must be greater than the refractive index of the capillary 1.tself. Teflon has a refractive index in the range of 1.35-1.38 and has been well characterized in zone electrophoresis.12 Although the refractive index of water i.s only 1.3313, much less than that of teflon, organic modifiers 14 can be added to the buffer to raise the refractive index above 1.38 and may actually enhance electrophoretic resolution. We chose ethylene glycol as an additive because of its high refractive index enhancement per percent added. The liquid medium naturally must remain ionic for proper electrophoresis.

Figure 4 shows that the same two test compounds can be separated in the ethylene glycol/water based buffer. The key ingredient in the separation buffer is $50 \mathrm{mM}$ sodium 
Figure 4. Electropherogram of bromothymol blue and bromocresol green separated in a Teflon capillary (50 $\mathrm{mm}$ 1.d.). Buffer: 508 by volume ethylere glycol, $10 \mathrm{ml}$ phosphate, $50 \mathrm{mM}$ SDS adjusted to pH 7.6. Bromothymol blue concentration: $5 \times 10^{-5} \mathrm{M}$. Bromocresol green concentration: $2 \times 10^{-5} \mathrm{M}$. Applied potential: +20 kv. Infection time: $20 \mathrm{~s}$. Effective time constant was $20 \mathrm{~s}$. 


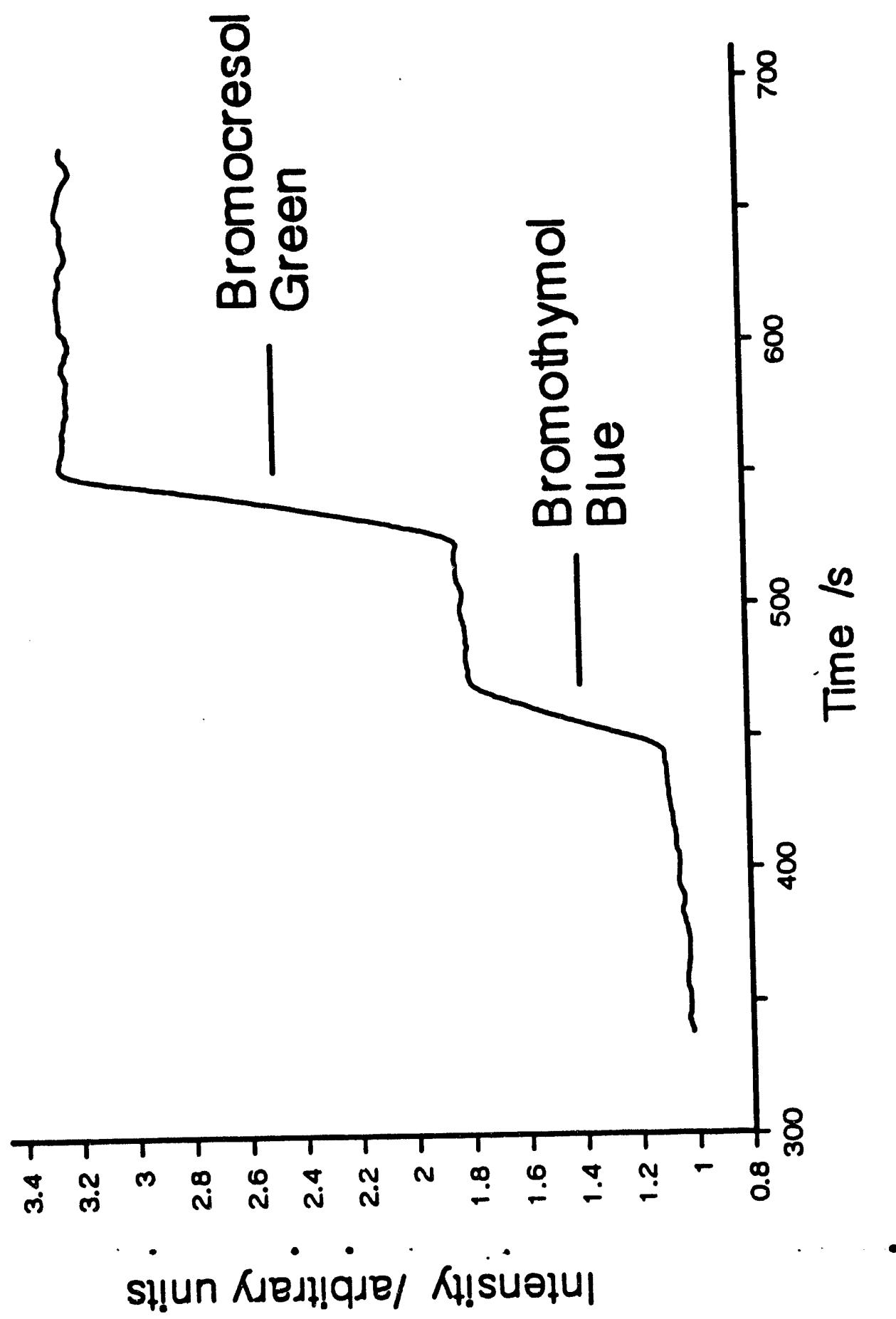


dodecyl sulfate (SDS).15 our explanation of the role of SDS is as follows: the presence of the organic modifier favors ion pair formation for the two test compounds. These ion pairs tend to interact with the teflon capillary causing band smearing. SDS micelles provide a moving organic phase which carries the analyte ion pairs along and gives each of the compounds a distinct mobility based on its affinity for the micellar phase. SDS ions also adsorb to the teflon walls producing a zeta potential and generating electroosmotic flow similar to that seen in silica capillaries. The evidence for enhanced electroosmotic flow is that, in the presence of SDS, analyte flow is directed toward the cathode, while in its absence, this flow is directed toward the anode.

Detection limits. The main source of noise limiting the signal-to-noise ratio of the transmitted light beam is capillary vibration. These effects of capillary vibration have been discussed earlier, 9 and in the present case, vibration arises from electrostatic motion in the very large applied potential field. With the teflon capillary glued onto a glass support and by applying a digital smoothing routine 10 with an effective time constant of about five. seconds, the signal-to-noise ratio measured oyer. 20 seconds (one peak width) is 600 for the transmitted 
beam. This gives an absorbance limit of detection (ALOD at $s / N=3$ ) of $2 \times 10^{-3}$ a.u.

In order to compare our results to the performance of the common cross-beam absorbance detector, we must use a criteria which is independent of analyte molar absorptivity. This can be accomplished by using the ratio of the absorbance limit of detection to the absorbance pathlength $(A L O D / b)$. For a typical cross-beam system, 16 the ALOD was $5 \times 10^{-4}$, resulting in $A L O D / b=1 \times 10^{-2}$

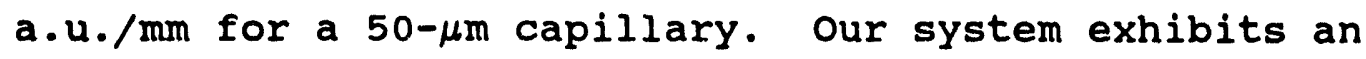
ALOD/b of $7 \times 10^{-4} \mathrm{a} . \mathrm{u} . / \mathrm{mm}$, a 15 fold improvement over the cross-beam arrangement. It is also important to note that if the capillary diameter is decreased further, such as in the case when reduced Joule heating is needed to maintain a high separation efficiency, there will be a proportionate increase in this improvement factor. Note that we are comparing an integral electropherogram with the standard electropherogram. As pointed out earlier, 9 integration of standard electropherograms is not always possible because of drifting baselines.

In summary, we have shown that an axial-beam absorption detection mode is feasible for CE. Even though a. laser is used hẹre for ease of coupling to the capillary tube, there is no reason why a conventional light source 
cannot be used. For example, the commercial absorption detectors for CE are already capable of collimating light

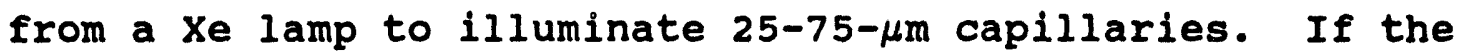
capillary can be held rigidly, then the higher stability of conventional light sources can even translate into better detectability. As discussed earlier, 11 the present detection mode has a limited dynamic range at high concentrations because of depletion of transmitted light, but the lower concentration range is extended because of better detectability. 


\section{ACRNOWLEDGEMENTS}

The authors wish to thank L. Koutny for operating the CCD camera. The Ames Laboratory is operated by Iowa state University for the U.S. Department of Energy under Contract No. W-7405-Eng-82. This work was supported by the Director of Energy Research, Office of Health and Environmental Research. 


\section{REFERENCE8}

1. S. Hjerten, J. Chromatogr., 347 (1985) 191.

2. Y. Walbroel and J. W. Jorgenson, J. Chromatogr., 315 (1984) 135.

3. Z. Prusik, V. Kasicka, S. Stanek, G. Kuncova, M. Hayer and J. Vrkoc, J. Chromatogr., 390 (1987) 87.

4. X. Huang, W. F. Coleman and R. N. Zare, J. Chromatogr., 480 (1989) 101

5. W. G. Kuhr, Anal. Chem., 62 (1990) 404R.

6. K. Fuwa, W. Lei and K. Fujiwara, Anal. Chem., 56 (1984) 1640.

7. W. Lei, K. Fujiwara and K. Fuwa, Anal. Chem., 55 (1983) 951.

8. K.-I. Tsunoda, A. Nomura, J. Yamada and S. Nishi, Appl . Spectrosc., $44(1990) 163$.

9. X. Xi and E. S. Yeung, Anal. Chem., 62 (1990) 1580.

10. A. Savitzky and M. J. E. Golay, Anal. Chem., 36 (1964) 1627.

11. R. E. Synovec and E. S. Yeung, Anal. Chem., 57 (1985) 2162.

12. K. D. Lukacs and J. W. Jorgenson, J. High Resolut. Chromatogr. Chromatogr. Commun., 8 (1985) 407.

13. R. C. Weast, M. J. Astle and W. H. Beyer (Editors), CRC Handbook of Chemistry and Physics, 65th ed., CRC Press, Boca Raton, 1984, p. E-357.

14. S. Fujiwara and S. Honda, Anal. Chem., 59. (1987). 487. 
15. S. Terabe, K. Otsuka and T. Ando, Anal. Chem., 57 (1985) 834.

16. N. J. Dovichi, F. Zarrin, T. G. Nolan and D. J. Bornhop, Spectrochimica Acta, 43B (1988) 639 . 
PAPER 2. AXIAL-BEAM LASER-EXCITED FLUORESCENCE DETECTION IN CAPILLARY ELECTROPHORESIS 


\author{
AXIAL-BEAM LASER-EXCITED FLUORESCENCE \\ DETECTION IN CAPILLARY ELECTROPHORESIS \\ John A. Taylor and Edward S. Yeung* \\ Department of Chemistry and Ames Laboratory-USDOE \\ Iowa state University, Ames, IA 50011
}

Reprinted with permission from Analytical Chemistry 1982 , 64, 1741-1744. Copyright 1992 American Chemical society. 


\section{AB8TRACT}

An approach for coupling a laser beam axially into a capillary for fluorescence detection in capillary electrophoresis is reported. The laser beam is focused into a small optical fiber which is in turn inserted into the separation capillary. Fluorescence is collected perpendicular to the capillary axis from a region located 5-10 mm from the end of the excitation fiber. Light propagation along the capillary axis under total internal reflectance conditions reduces light scatter through the capillary walls and allows the use of capillaries with intact silicone or polyimide coatings. Concentration detection limits for Rhodamine $6 \mathrm{G}$ are in the $10^{-11} \mathrm{M}$ or lower range. 
INTRODUCTION

Capillary electrophoresis (CE) has emerged as a powerful separations technique, with applications from simple atomic ions ${ }^{1}$ to large DNA fragments. 2 A challenging problem is detection and monitoring of the analytes after they are separated, since sub-nanoliter volumes are involved. Laser-excited fluorescence detection so far has provided the lowest detection limits, down to the few-hundred molecules range. ${ }^{3}$ These low detection limits are essential for applications like DNA sequencing, 4 where the sample sizes must be kept small to avoid clogging up the gels. Laser-excited fluorescence detection typically requires careful alignment both in excitation (to couple with the small i.d. of the capillaries) and in light collection (to discriminate against stray light). Also, the standard geometry for excitation perpendicular to the axis of the capillary requires the creation of an optically clear region in the polyimide coating. This makes the capillary fragile and complizates the preparation of capillaries for use. Furthermore, the excitation pathlength, hence the fluorescence signal, is restricted to the small diameter of the capillary. 
One approach to increasing the excitation pathlength is to direct light along the capillary rather than across it. Capillary electrophoresis detection along the capillary axis has been studied extensively. In our group, axial-beam absorbance detectors ${ }^{5-7}$ have been developed which utilize total internal reflection of the incident light beam. Sweedler et al.8 have designed a fluorescence detector with axial-beam excitation and CCD detection for signal enhancement by integration and spectral analysis. The main difference in that arrangement is that light is propagated through the capillary by partial internal reflection. Total internal reflection is advantageous in fluorescence excitation because all of the light entering the capillary within a certain critical angle will propagate with little loss. This results in less noise due to capillary vibrations (misalignment) and a detector response that should be independent of the location of the analyte band. The capillary can thus be bent without attenuating the excitation beam. An added advantage in axial-beam fluorescence excitation with total internal reflection is that there is very little (in principle, none) scattered light originating from the capillary walls. This should allow the use of capillaries with intact polyimide coatings without problems of interference due to 
absorption or greatly increased fluorescence background. Another benefit from the axial-beam geometry is a longer absorption pathlength compared to irradiation across the capillary. Unlike axial-beam absorption, 5-7 however, the observation length here must be small compared to the analyte zone length. Still, this implies increasing the interaction length from $75 \mathrm{\mu m}$ to $1 \mathrm{~mm}$ to provide increased fluorescence intensity. Finally, axial-beam illumination opens up the possibility of whole-column detection. 9 The purpose of this paper is to demonstrate the feasibility of axial-beam fluorometry for CE. 


\section{EIPERIMENTAL BECTIOA}

A schematic of our fluorescence detector is shown in Figure 1 .

Excitation. The light source for excitation was an air-cooled argon ion laser (Uniphase, San Jose, CA, model 2213-150ML) operating at several visible lines which were separated externally using a glass prism. The $514 \mathrm{~nm}$ line was used for excitation at a power of $5 \mathrm{~mW}$ (a fraction of the $14 \mathrm{~mW}$ all-lines output). A principal advantage of this laser is its intensity stability of $0.05 \%$ for all lines. Although stability for the isolated 514 line is in the one part in 200 range, this offers sufficient background stability. The beam was directed through two mirrors on adjustable mounts (Newport, Fountain Valley, CA) and focussed using a $10 \mathrm{X}$ microscope objective (BM2888, $16 \mathrm{~mm}$, Bausch L Lomb, Rochester, NY) into an optical fiber (\#B0-0185-10, Fiberguide Industries, stirling, NJ, core diameter $=46 \mu \mathrm{m}$, cladding $=50 \mu \mathrm{m}$, jacket $=51 \mu \mathrm{m})$. Transmission and coupling losses reduced the power throughput of the fiber to about 408 of that in the incident laser beam. The fiber was inserted, by hand, either 1.0 or $1.5 \mathrm{~cm}$ into the separation capillary of 


\section{Figure 1. Schematic for fluorescence detection with axial}

illumination. 


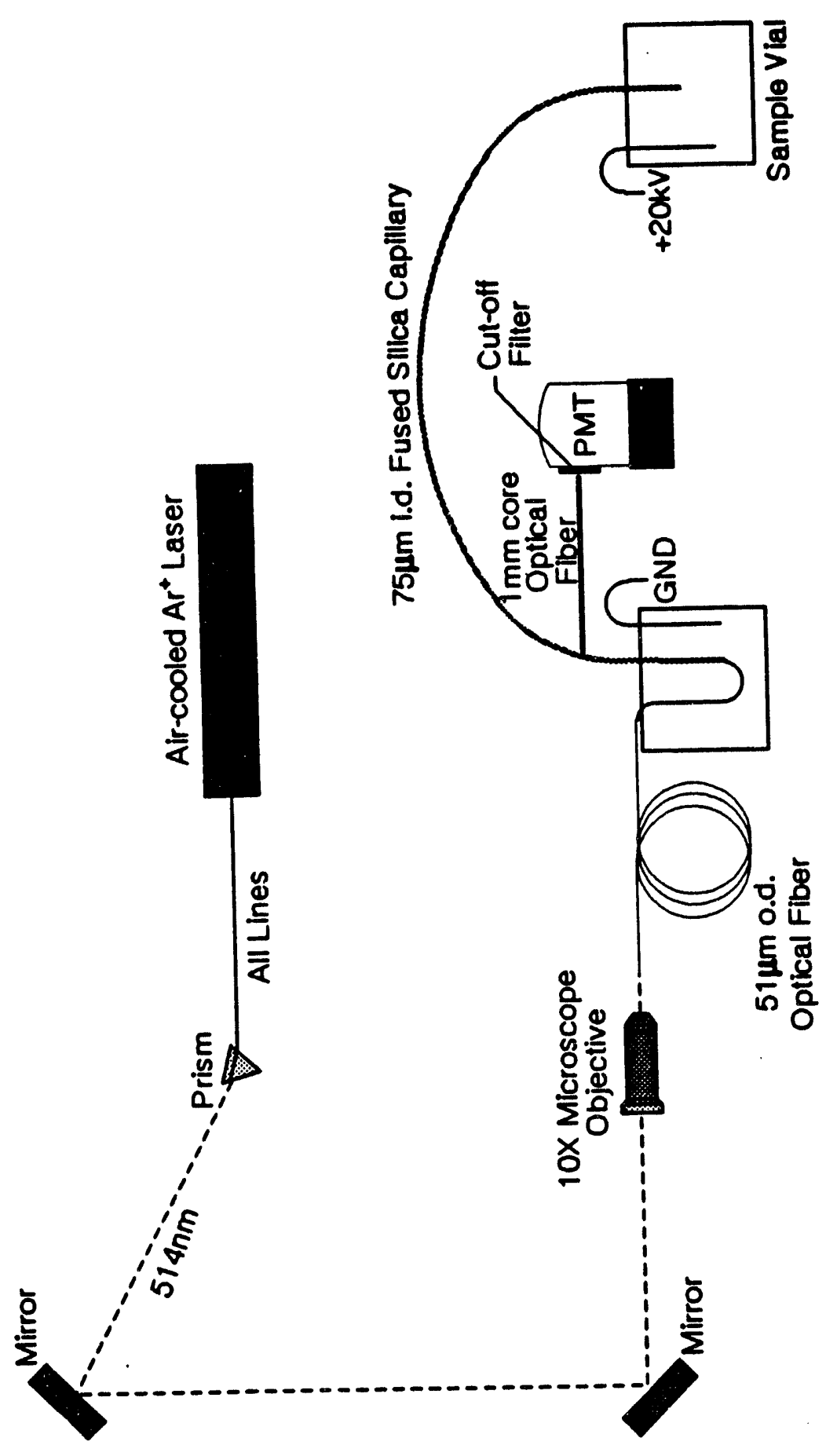


$75 \mu \mathrm{m}$ i.d. This process is fairly easy to perform after some practice.

Detection. A larger optical fiber (core diameter $=1$ $\mathrm{mm}$ ) was placed perpendicular to the capillary wall, either 5 or $10 \mathrm{~mm}$ above the end of the excitation fiber, or $2 \mathrm{~cm}$ from the exit end of the capillary. This 1-mm fiber was used to collect fluorescence emission from the capillary and transmit it through two cutoff filters (0G570, Melles Griot, Irvine, $C A$ ) held flush against the photomultiplier tube surface (Hamamatsu, Middlesex, NJ, R928, operating voltage $=900 \mathrm{~V})$. The resulting current signal was amplified using a picoammeter (Keithley, Cleveland, $O H$, Model 417) and displayed on a chart recorder.

separation. A high refractive-index capillary electrophoresis solvent was prepared by mixing $1 \mathrm{ml} 0.05 \mathrm{M}$ $\mathrm{NaCl}$ (Fisher, Fair Lawn, NJ, Certified ACS Grade) in water per $100 \mathrm{ml}$ dimethyl sulfoxide (Fisher, Certified ACS). Capillaries with $75-\mu \mathrm{m}$ i.d. (Polymicro Technologies, Phoenix, AZ; polyimide coated, \#TSP075150; silicone coated, \#TSS075170) were used without removing the protective coating in the detection region. Electrokinetic injection at $20 \mathrm{kV}$ was carried out by momentary activation (4 s) of a high voltage power supply (Glasman, Whitehouse station, NJ, model PS/MJ30P0400-11) using a homemade timer circuit. 
Serial dilutions of DOCI (Exciton, Dayton, $\mathrm{OH}$ ) and Rhodamine 6G (Allied Chemical, Morristown, NJ) were prepared from $10^{-6} \mathrm{M}$ stock solutions and were separated at $20 \mathrm{kV}$ applied potential over a $30 \mathrm{~cm}$ length of capillary. Capillary image. Images of $75-\mu \mathrm{m}$ i.d. capillaries were taken through a microscope (Bausch and Lomb, Stereo Zoom 7) and recorded using a CCD camera (Photometrics, Tucson, AZ, Series 200). Two $2 \mathrm{~s}$ exposures were taken with a 3 s interval between them. In order to measure particle migration rates in this small viewing length, the potential across the capillary was reduced to $500 \mathrm{~V}$. 


\section{RESULTS AND DIBCUSBION}

General considerations. In our previous reports on axial-beam absorption detection, 5-7 the laser beam was simply focused at the liquid inlet of the capillaries. This was possible because the transmitted intensity through the liquid core, which contains the analytical information, is much larger than that transmitted through the fused-silica capillary walls. The polyimide coating actually helps to distinguish between the two light paths, since it absorbs the laser beam slightly at each reflection. In fluorescence detection, however, the capillary walls and the polyimide coating fluoresce if they are irradiated by the laser light. This can overwhelm the small fluorescence signal from the analytes. It is therefore desirable to focus the excitation laser beam into an optical fiber, which in turn is inserted into the separation capillary. Because of the limited aperture of the optical fiber, light is efficiently coupled to the liquid core, provided that the exit face of the optical fiber is cleanly cleaved and is perpendicular to the axis. Such an interface is also advantageous because alignment of the optical fiber with the laser beam can be made permanent. Changing the separation capillary is then 
simplified, and at the $50-\mu \mathrm{m}$ level alignment does not even require the use of a microscope. We note that single-mode optical fibers can be made with diameters down to $5 \mu \mathrm{m}$. So coupling with capillaries at these dimensions and larger is possible.

Detection of fluorescence in the present work is through a large-diameter optical fiber perpendicular to the capillary. Even though excitation is in the axial direction, only a fraction of the eluted bands is viewed at any one time. The electropherograms therefore resemble conventional ones and not the integral electropherograms in the analogous absorption scheme. 5 The observation region must then be short compared to the analyte zone lengths to avoid band-broadening. The longer the observation region, however, the larger the fluorescence intensity. The present arrangement represents such a compromise. In principle, if the observation region is well defined, deconvolution using procedures similar to those applicable to finite slit widths in spectroscopy can restore all of the electrophoretic information. Naturally, it is also possible to use a mechanical slit to define the observation region and still allow easy and reproducible alignment, since the slit is across the length of the capillary and not across its width. 
The insertion of an optical fiber at the end of the capillary minimizes contributions from the surfaces of the optical fiber to the separation. There is still a slight change in electroosmotic flow rate because of a changing zeta potential. There can also be a slight pressure differential because of the restricted cross-section. So, the overlap region should be made short compared to the length of the capillary. The requirement that total internal reflection is achieved limits the choice of the electrophoretic buffer here. However, materials other than fused silica can be used for the separation capillary so that buffers with much lower refractive indices become applicable. 6,10

Photochemical bleaching. Increased laser power should also be advantageous in providing a larger analyte signal. However, fluorophores are easily bleached by the laser beam, even at the $\mathrm{mW}$ range, negating any increase in excitation intensity. Furthermore, in the axial-beam arrangement, fluorophores are quite likely completely bleached before they reach the observation region simply because of the residence time in the excitation beam. A novel way to increase the incident laser power without bleaching the compounds of interest is to add an inert absorber to the solvent (buffer) contained in the 
capillary. In a capillary containing an absorber producing an absorbance of 0.6 per $\mathrm{cm}$ of pathlength, for example, the incident laser intensity is only reduced to $50 \%$ in $5 \mathrm{~mm}$, the distance between the end of the optical fiber and the observation region. But over a distance $4 \mathrm{~cm}$, it is reduced to less than $0.5 \%$ of its original value. Thus if a detection device is placed close to the point where excitation light is introduced, an analyte band traveling from the opposite end of the capillary will interact with very little light until it gets close to the detection region. It is necessary to isolate the observation region from the end of the optical fiber, however, so that specular reflections and fluorescence from the fiber can be discriminated against. There is a possibility that energy transfer or even quenching exists between the analyte and the absorber.11 so the absorber has to be carefully selected to maintain quantitative reliability.

As mentioned above, an ionic absorber, orange G, at $4 \times 10^{-5} M$ has been added to address the problem of photobleaching. The electropherograms in Fig. 2 illustrate the effectiveness of this addition. Fig. 2 a shows the separation of two fluorescing dyes, 3,31-diethyloxacarbocyanine iodide (DOCI) and Rhodamine 6G. The excitation beam had been blocked off for most of the 


\section{Figure 2.}

Separation of DOCI (1) and Rhodamine 6G (2) in silicone coated capillary. (a) The excitation beam has been blocked for the first $4.75 \mathrm{~min}$ of the run. (b) The excitation beam has been blocked for the first $5.75 \mathrm{~min}$ of the run. (c) The inert absorber, $20 \mu \mathrm{M}$ orange G, has been added. The excitation beam remains unblocked through the entire run. (d) orange G concentration has been increased to $40 \mu \mathrm{m}$, but other conditions are identical to those in (c). 

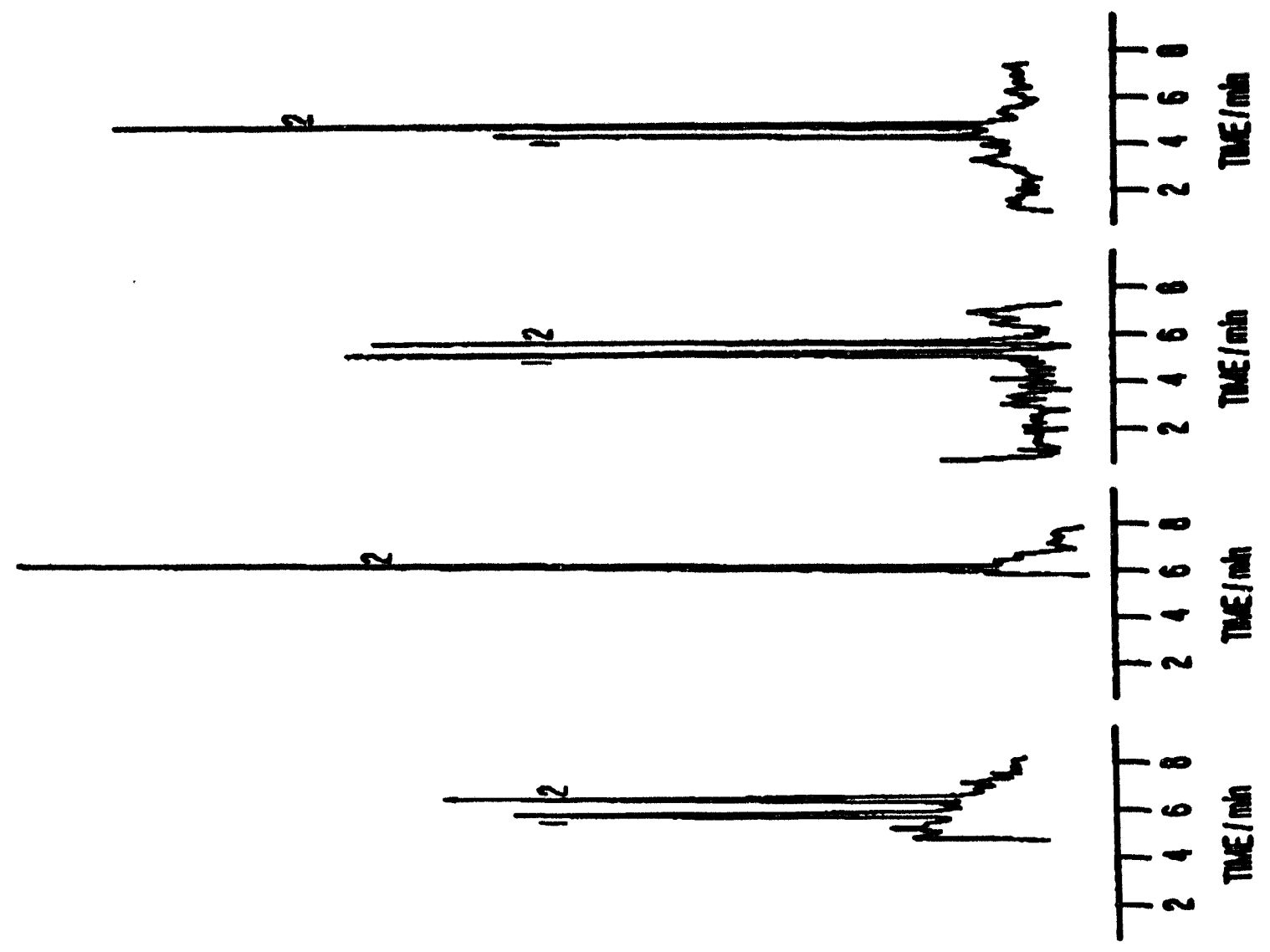
migration time along the capillary to prevent complete bleaching of the analyte bands, and was unblocked slightly before the appearance of the first compound at the detection zone. DOCI is a much weaker absorber at $514 \mathrm{~nm}$ and is less prone to photochemical bleaching than Rhodamine 6G. This portion of the figure shows roughly the same peak height for both dyes. In Fig. $2 b$, the laser has been blocked until just before the emergence of the second peak, yielding a Rhodamine $6 \mathrm{G}$ signal which is almost twice as large as that seen in Fig. $2 a$.

Fig. 2c shows the same separation carried out with $20 \mu \mathrm{M}$ Orange $\mathrm{G}$ in the running buffer (absorbance $=0.3$ per $\mathrm{cm}$ of pathlength). The slight difference in retention times is a function of capillary surface variations rather than the presence of the inert absorber. Note that the Rhodamine $6 G$ peak is comparable in size to the DOCI peak. When the concentration of orange $G$ is raised to $40 \mu \mathrm{M}$ (Fig. 2d), the relative peak height is roughly twice that of DOCI, indicating that bleaching has been reduced to a level similar to that seen in Figure $2 \mathrm{~b}$. The orange $G$ anion, which is continually replenished by its electrokinetic mobility, eliminates the need to block and unblock the laser beam and provides a consistent mode for separation. There is of course no longer uniform illumination along the 
capillary, but since the observation point is fixed, quantitation should not be a problem. If necessary, Beer's Law can be applied since the distances are clearly defined.

Capillary coatings. As mentioned earlier, one distinct advantage of the axial-beam technique is that it allows the supportive coating on the capillary to remain intact. Most fused silica capillaries have a polyimide coating known to emit a broad fluorescence when exposed to wavelengths of light under $600 \mathrm{~nm}$. If a through-the-wall excitation scheme is used without first removing this coating the fluorescence background will completely overtake the weak analyte signal at low concentrations. For axial excitation with total internal reflection, all of the light remains inside the capillary and generally cannot escape to the outside coating. Our experience is that the fluorescence background observed with polyimide coated capillaries is no more than twice that seen with capillaries coated with non-fluorescing polymers. The polyimide contribution to fluorescence in this case can be explained by micro imperfections in the capillary surface allowing small amounts of light to breach conditions of total internal reflection.

To demonstrate the feasibility of detection through polymer coatings, Figures 3 and 4 respectively show the 
59

Figure 3. Separation of DOCI (1) and Rhodamine $6 G$ (2) in silicone coated capillary. In this case, the excitation fiber has been placed $0.5 \mathrm{~cm}$ from the detection region instead of $1 \mathrm{~cm}$ as in Fig. 2 . 
60
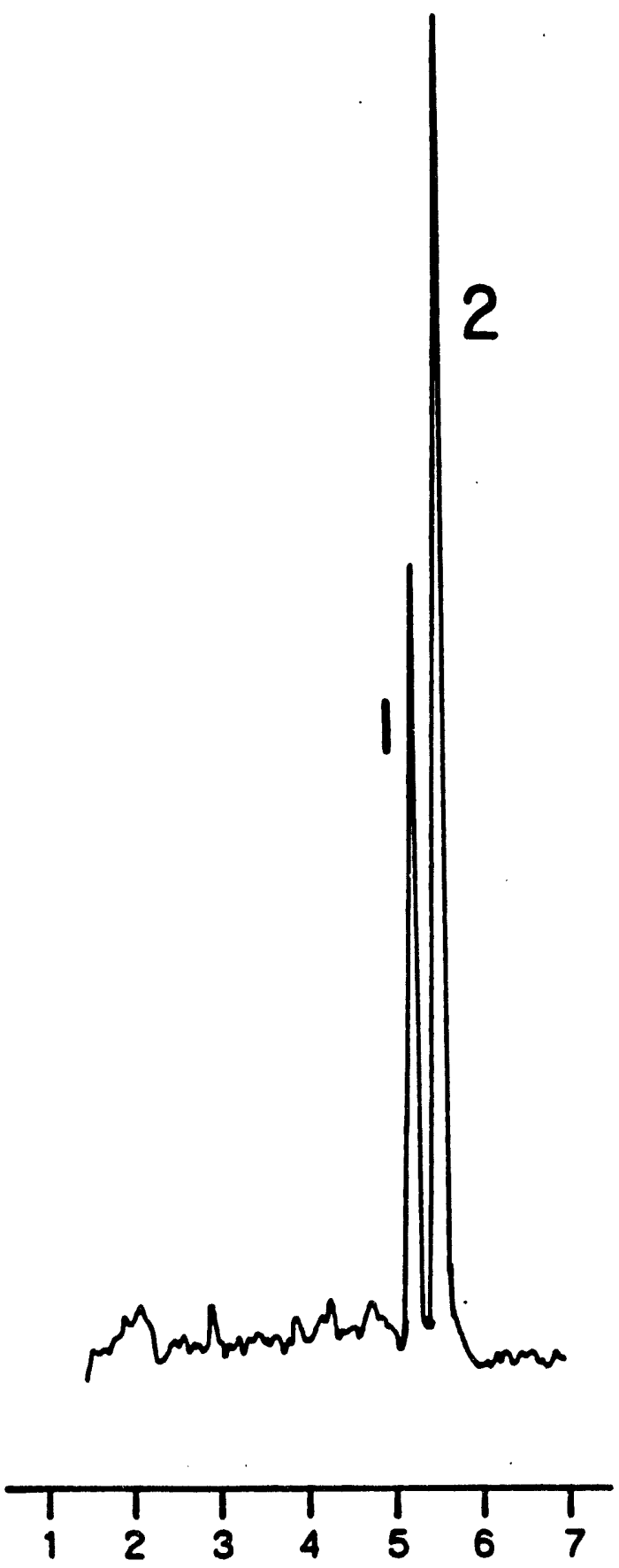

TIME / $\mathrm{min}$ 
Figure 4. Separation of DOCI (1) and Rhodamine 6G (2) in polyimide coated capillaries. The unnumbered peaks are caused by light scattered by sub-micron sized particles flowing through the detection region. 


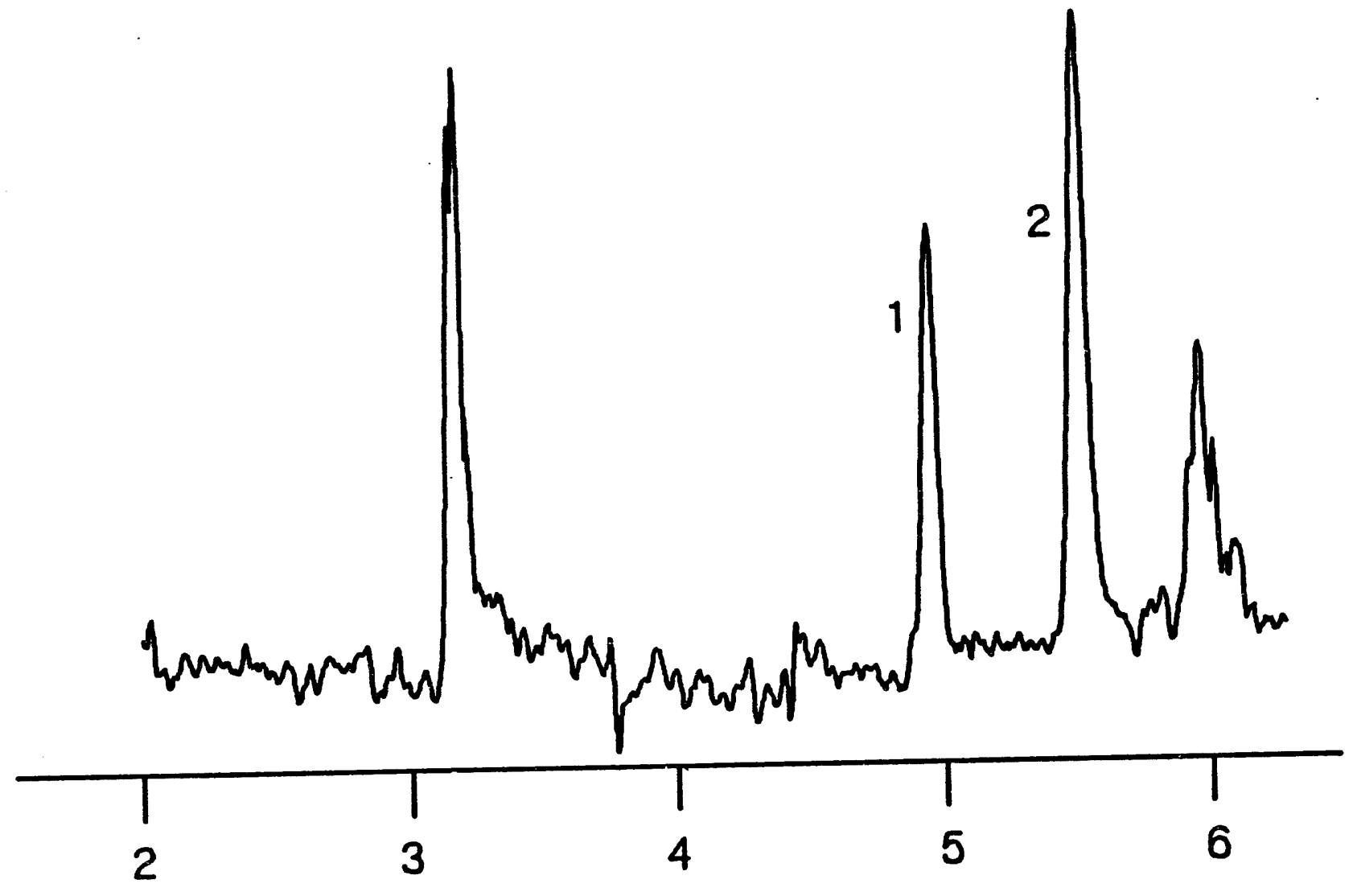

Time / $\mathrm{min}$ 
separation of $10^{-8} \mathrm{M}$ DOCI and $10^{-10} \mathrm{M}$ Rhodamine $6 \mathrm{G}$ in capillaries with non-fluorescing silicone and fluorescing polyimide coatings. Based on these figures, concentration detection limits $(S / N=3)$ for rhodamine $6 G$ are estimated to be $6 \times 10^{-12} \mathrm{M}$ and $1 \times 10^{-11} \mathrm{M}$ respectively. The detection limit with polyimide coating is slightly higher because of the noise contribution of the background fluorescence. These concentration levels are not among the lowest reported, 3 but are comparable to typical values obtained in laser-excited fluorometric detection.12 The interference spikes seen in Figure 4 are the result of sub-micron sized particles in the solvent. These particles scatter excitation light through the capillary walls, producing fluorescence from the polyimide coating. Because of their size, the particles are almost impossible to remove by filtration, but their effects are easily discriminated against because their response time (observed peak width) is short compared to the typical sample plug. They also do not show a monotonic increase and decrease in response, as is evident in Fig. 4. The ratio of the response times can be estimated from the ratio of the observation length for a particle ( $\mathrm{mm}$ here) to the analyte zone length (approximately $5 \mathrm{~mm}$ ). 
Noise characterization. Since the presence of microparticles can interfere with quantitation and degrade detection limits for the analytes, we proceeded to further characterize them. There appears to be no decrease in the number of such scattering events whether or not the solutions were filtered (through $0.4-\mu \mathrm{m}$ filters) and/or degassed. This shows that the scattering centers are solid particles of sub-micron diameters. This is further confirmed by examination in a microscope while the capillary is axially illuminated. At a resolution of $1 \mu \mathrm{m}$, the scattering centers are still point-like with no noticeable cross-sections. CCD images of such a capillary under simulated electrophoresis conditions through a microscope are shown in Figure 5. Spots from dust particles and imperfections in the polyimide coating are evident. Generally these are stationary, as confirmed by the consecutive pictures in Fig. $5 \mathrm{a}$ and $5 \mathrm{~b}$. These do not contribute directly to background noise in the normal cross-beam excitation geometry, as long as the 10-50 $\mu \mathrm{m}$ laser beam waist does not overlap them. They do however add to the background level and thus noise in the axial beam arrangement here because the observation length is about $1 \mathrm{~mm}$. In both Fig. $5 \mathrm{a}$ and $5 \mathrm{~b}$, one can observe streaks of scattered light oriented along the capillary. 


$$
65
$$

Figure 5. Images of scattering centers inside a capillary during electrophoresis. Streaks (1 and 2) indicate motion of two particles during the $2 \mathrm{~s}$ exposures. Since the elapsed time between frames $a$ and $b$ is only $3 \mathrm{~s}$, the label 1 corresponds to the same particle in both frames. The imaged length is about $0.5 \mathrm{~mm}$. 
a

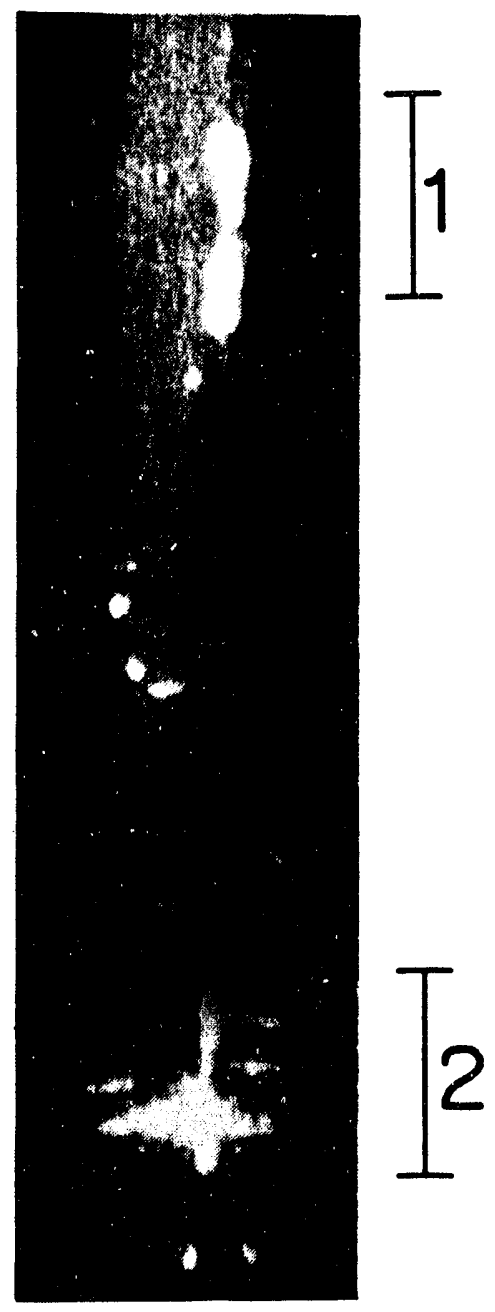

b

Flow

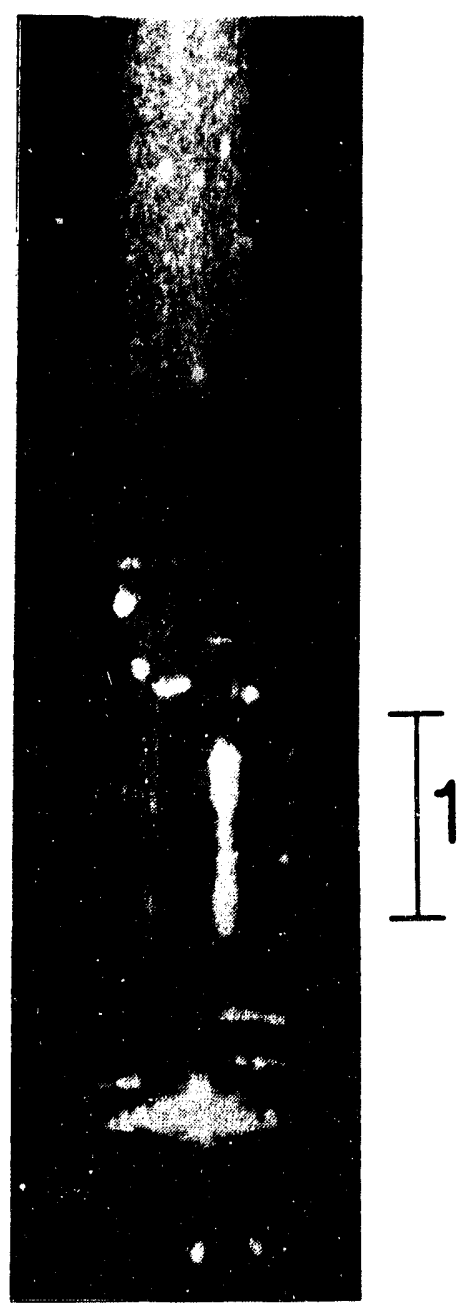


These represent particles moving along the capillary during the 2-s exposure time. The time delay between these successive images is consistent with the conclusion that the same particle was imaged in both frames. The length of the streak is consistent with the estimated electroosmotic (EO) flow rate at these running conditions. In fact, this may serve as a good alternative to directly monitor EO flow rates, if particles that are not expected to acquire much charge/mass and thus finite electrophoretic mobility are deliberately introduced into the system. It is interesting to note that the streaks are at different positions across the capillary, confirming bulk flow. The streaks also fade in and out in each image, depicting a tumbling motion in and out of focus. This explains the structures on the scattering peaks in Fig. 4. Finally, it is clear that such particles also affect the normal cross-beam geometry for fluorescence excitation. The smaller the laser beam waist, the shorter is the particle residence time and the lower is the probability that the particle actually traverses the part of the capillary cross-section that contains the laser beam. This explains why the overall particle scattering effect is less pronounced in standard laser-excitation geometries. The effect however is definitely present, as 
definitely present, as supported by numerous such observations in our own laboratory.

The use of DMSO here is dictated by the need to overcome the refractive index of fused silica to provide total internal reflection. For excitation in the visible region, the background fluorescence level appears to be no different than that from purified water. The number of scattering centers (particles) per unit time is also similar. As we have demonstrated previously, 6 a water ethylene-glycol mixture can serve the same purpose if teflon capillaries are used instead. The introduction of the absorber does create additional fluorescence background. We estimate the fluorescence quantum yield of orange $\mathrm{G}$ to be $10^{-4} .11$ This then is equivalent to having a nM concentration of a good fluorophore in the system. With the laser intensity stabilized to the $0.1 \%$ level, noise equivalent to $\mathrm{pM}$ analyte can be expected. 


\section{ACRNOWLEDGEMENTS}

The authors thank Lance Koutny for setting up the CCD imaging system. Ames Laboratory is operated for the U. $S$. Department of Energy by Iowa State University under contract No. W-7405-Eng-82. This work was supported by the

Director of Energy Research, Office of Health and Environmental Research. 


\section{LITERATURE CITED}

1. Gross, L.; Yeung, E. S. J. Chromatogr, 1989, 480, 169-178.

2. Cohen, A. S.; Najarian, D. R.; Smith, J. A.; Karger, B. L. J. Chromatogr. $1989,458,323-333$.

3. Cheng, Y. F.; Dovichi, N. J. Science 1988, 242, 562-564.

4. Cohen, A. S.; Najarian, D. R.; Karger, B. L. J. Chromatogr. $1990,516,49-60$.

5. Xi, X.; Yeung, E. S. Anal. Chem, 1990, 62, 1580-1585.

6. Taylor, J. A.; Yeung, E. S. J. Chromatogr. 1991, 550, $831-837$.

7. Xi, X.; Yeung, E. S. Appl. Spectrosc. 1991, 45, 1199-1203.

8. Sweedler, J. V.; Shear, J. B.; Fishman, H. A.; Zare, R. N.; Scheller, R. H. Anal Chem. 1991, 63, 496-502.

9. Rowlen, K. L.; Duell, K. A.; Avery, J. P.; Birks, J.W. Anal. Chem. 1989, 61, 2624-2630.

10. Tsunoda, K.; Nomura, A.; Yamada, J.; Nichi, S. Appl. Spectrosc. $1990,44,163-165$.

11. Garner, T. W.: Yeung, E. S. Anal. Chem. 1990, 62, 2193-2198. 12. Kuhr, W. G. Anal. Chem. 1990, 62, 403R-414R. 
71

PAPER 3. MULTIPLEXED FLUORESCENCE DETECTOR FOR CAPILLARY ELECTROPHORESIS USING AXIAL OPTICAL FIBER ILLUMINATION 
Multiplexed Fluorescence Detector for Capillary Electrophoresis Using Axial optical Fiber Illumination

John A. Taylor and Edward S. Yeung*

Ames Laboratory-USDOE and Department of Chemistry

Iowa State University, Ames, IA 50011

Reprinted with permission from Analytical Chemistry 1993, 65, 956-960. Copyright 1993 American Chemical Society. 


\begin{abstract}
A fluorescence detector for capillary electrophoresis with the ability to simultaneously monitor separations in multiple capillaries in real time is described. This multiplexing approach involves laser illumination of a bundle of optical fibers which are inserted into a capillary array. The array is imaged orthogonally through a microscope onto a charge-coupled device camera for signal analysis. The technique is demonstrated for ten capillaries and considerations for upscaling to as many as one thousand capillaries are discussed.
\end{abstract}




\section{INTRODUCTION}

It is obvious that irrespective of whichever basic technology is eventually selected to sequence the entire human genome, 1,2 there are substantial gains to be made if a high degree of multiplexing of parallel runs can be implemented. Such multiplexing should not involve expensive instrumentation and should not require additional personnel, or else the main objective of cost reduction will not be satisfied even though the total time for sequencing is reduced. A corollary is that if a certain basic technology can be readily: ficiently highly multiplexed, then it may ultimate scome the methoa of choice to sequence the entire human genome. In fact, present-day sequencing runs are already being performed in slab gels with multiple lanes to achieve multiplexing. Unfortunately, slab gels are not readily amenable to a high degree of multiplexing and automation. Difficulties include uniform gel preparation over a large area, reproducibility over different gels, loading of sample wells, large physical size of the medium, uniform cooling, large amounts of media, buffer, and samples needed, and long run times for extended reading of bases. The introduction of ultra-thin slab gels ${ }^{3}, 4$ does address some 
of these difficulties. Still, it is hard to imagine how 1,000 lanes can be run simultaneously in one instrument. In the last two years, several research groups have shown that capillary gel electrophoresis (CGE) is an attractive alternative to slab gel electrophoresis (SGE) for DNA sequencing. 5-8 The medium used, buffer composition, separation mechanism, sequencing chemistry, and tagging chemistry for CGE are all derived from proven SGE schemes. A 25-fold increase in the sequencing rate per capillary (per lane) has already been demonstrated. This is a direct consequence of the small internal diameter of the capillary tubes, typically around 50-75 $\mu \mathrm{m}$, greatly reducing Joule heating associated with the electrical current. Gel distortions and temperature gradients that can affect resolution of the bands are thus virtually absent. More importantly, much higher electric fields can be applied to speed up the separation. For comparison, conventional SGE are limited to field strengths below 50 V/Cm while CGE have been successfully used for sequencing up to $500 \mathrm{~V} / \mathrm{cm} .7$ The unique aspect ratio of capillary gels (25-50 cm long) provides uniform field strengths and the large surface-to-volume ratio favors efficient heat removal. These combine to produce much sharper bands than are possible in slab gels. 
Part of the improvement in sequencing speed in CGE is counteracted by the inherent ability of slab gels for accommodating multiple lanes in a single run. So, unless capillary gels can be highly multiplexed and run in parallel, the above-mentioned advantages cannot lead to real improvements in sequencing the human genome. Fortunately, the capillary format is in fact well suited for multiplexing. Already mentioned is the substantial reduction of Joule heating per lane, even given the high applied fields, so that the overall cooling requirement and electrical requirement remain manageable. The cost of materials per lane is much reduced because everything, including sample sizes, is smaller. The reduced band dimensions are ideal for excitation by laser beams and for imaging onto solid-state array detectors. The use of electromigration injection provides reproducible sample introduction with little band spreading and with little effort. Commercial capillary electrophoresis instruments have already incorporated the necessary robotics for such an operation. The small band sizes however put stringent requirements on detection. It is not possible to use large amounts of DNA fragments because of overloading and plugging of the gel pores. 6 so, recently proposed multiplexing schemes such as multiple hybridization 8 and 
multiple tagging ${ }^{9}$ cannot be readily implemented in CGE. Parallel sequencing runs in a set of up to 24 capillaries have been demonstrated recently.10 To provide sensitive laser-excited fluorometric detection, a confocal illumination geometry couples a single laser beam to a single photomultiplier tube. Observation is one capillary at a time, and the capillary bundle is translated across the excitation/detection region at $20 \mathrm{~mm} / \mathrm{s}$ by a mechanical stage. This provides adequate observation time for each capillary (to achieve a reasonable signal-to-noise ratio for base calling) but fast enough to repeat the scan every second (to achieve reasonable temporal resolution). That study 10 clearly demonstrates that capillaries can be run in parallel for DNA sequencing.

There are some subtle features inherent to the confocal excitation scheme 10 that may limit its use for very large numbers (thousands) of capillaries. Since data acquisition is sequential and not truly parallel, the ultimate sequencing speed will be determined by the observation time needed per DNA band for an adequate signal-to-noise ratio. Having more capillaries in the array or being able to translate the array across the detection region faster will not increase the overall sequencing speed. That is, to achieve the same 
signal-to-noise ratio, if the state-of-the-art sequencing speed of 1000 bases/hr/lane 7 is used, the number of parallel capillaries ${ }^{10}$ will have to be reduced proportionately regardless of the scan speed. The use of a translational stage may become problematic for a large capillary array. It has been shown'l that bending of the capillaries can result in degradation in the separation efficiency. This is attributed to distortions in the gel and multipath effects. The amount of bending and cycling naturally increases with the number in the array. Sensitive laser-excited fluorescence detection also requires careful alignment both in excitation and in light collection, so that efficient coupling with the small i.d. tubing and discrimination of stray light are possible. 12 The translational movement thus has to maintain flatness to the order of the confocal parameter $10,13,14$ (around $25 \mu \mathrm{m}$ ) or else the cylindrical capillary walls will distort the spatially selected image. Multiple lenses and beamsplitters, beam scanning optics, or translational stages are therefore all unattractive. The latter two also have inherently a low duty-cycle which works against multiplexing.

Recently, we have developed an axial beam excitation scheme for capillary electrophoresis. 15 The excitation 
laser is coupled via an optical fiber which in turn is inserted into the capillary tube. Observation is outside the capillary walls perpendicular to the axis. In one mode of operation, total internal reflection of the excitation beam is achieved, so that no stray light from the quartz walls or the polyimide coating reaches the phototube. Axial-beam excitation also provides longer absorption/fluorescence pathlengths, increasing the signal. Detection at the $\mathrm{pM}$ level was demonstrated. We report here the use of this excitation geometry to simultaneously monitor 10 capillary tubes undergoing electrophoresis. This represents a truly parallel multiplexing scheme for monitoring large arrays of capillaries. The system has no moving parts. Data rates are compatible with state-of-the-art run times in CGE, and are fast enough even if there is another order of magnitude increase in DNA sequencing rates per lane in the future. 


\section{EXPERIMENTAL BECTION}

Excitation. A schematic diagram of the multiplexing apparatus is shown in Figure 1. Approximately 500 glass optical fibers (.002" 0.d., P31735, Edmond scientific, Barrington, $\mathrm{NJ}$ ) were grouped together and inserted into a 1/8" i.d. heat shrinking tube. After heating, a razor blade was used to cleave the heat shrink casing and the enclosed fibers. This fiber bundle was inserted into the laser beam path by a microscope objective (Bausch and Lomb, Rochester, NY $16 \mathrm{~mm}, 10 \mathrm{X}, \mathrm{BM} 2888$ ). The $488 \mathrm{~nm}$ laser beam (2011-30SL, Cyonics, San Jose, CA) was partially focused by the objective to provide a beam spot size that overlaps the desired number of fibers in the illumination zone. A total laser power of $5 \mathrm{~mW}$ was thus divided among 10-12 optical fibers. The free end of each illuminated fiber was inserted $1.5 \mathrm{~cm}$ into a different fused silica capillary as discussed previously.15

Detection. The capillary array in the detection region was imaged onto a charge-coupled device (CCD) camera (Photometrics, Tucson, AZ, Series 200) through the camera extension of a binocular microscope (Bausch and Lomb, stereo Zoom 7). The CCD camera was operated in a 25 to 1 column binning mode.16,17 This allowed compression of 200 columns and 375 rows of image data into a 8 × 375 array. 

CCD, charge-coupled device camera; AD, adapter column; MB, microscope body (eyepiece not shown); OF, optical fibers; SC, separation capillary; CC, capillary with black coating; MO, 10X microscope objective; GND, buffer reservoir at ground potential; HV, buffer reservoir at positive high potential. 


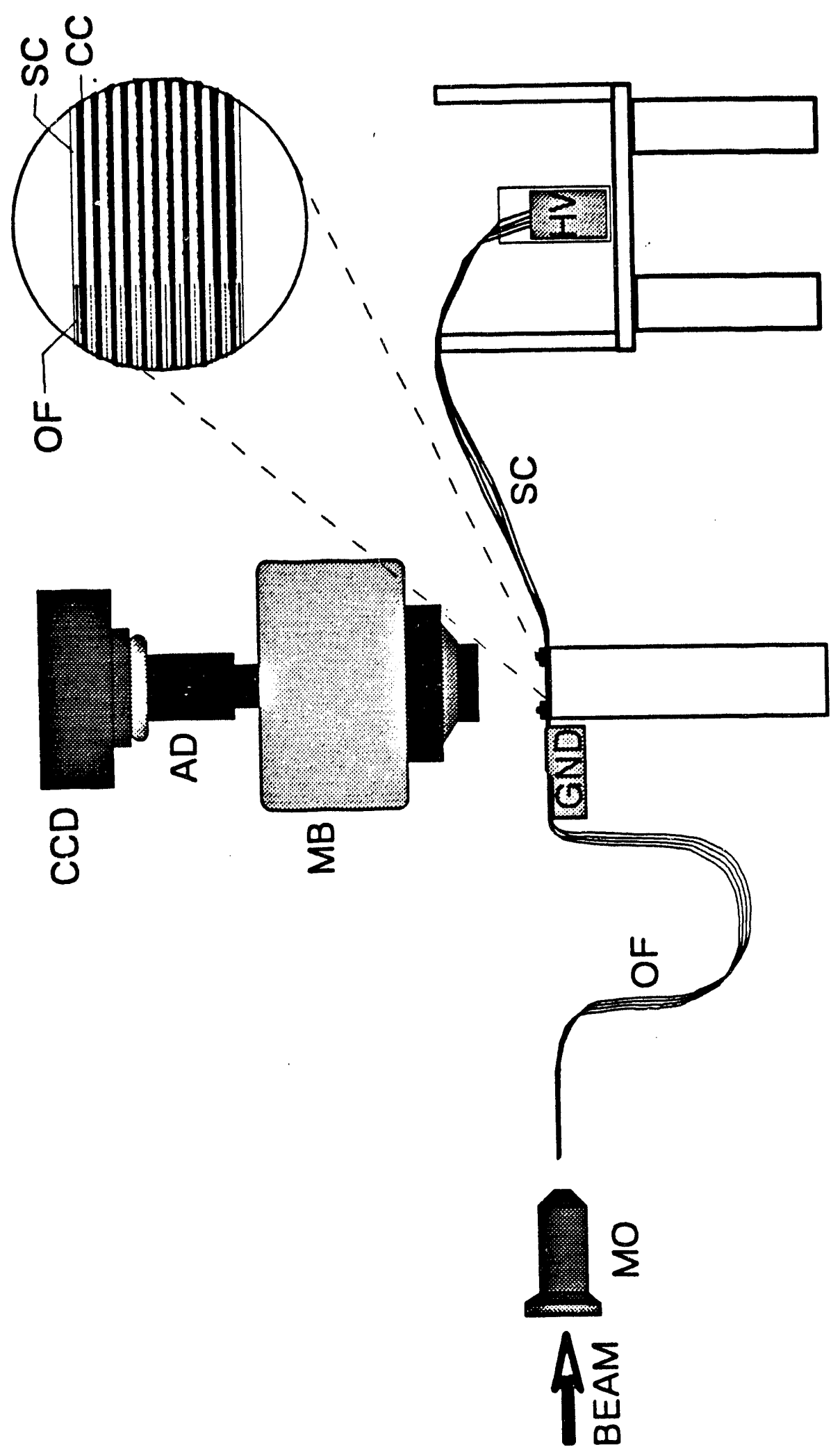


Data extraction from the $\mathrm{CC} 200$ memory and analysis was carried out on a PC-compatible 80386-based computer equipped with an IEEE interface. Each frame of data, corresponding to a $0.1 \mathrm{~s} C C D$ exposure taken every $0.9 \mathrm{~s}$, was stored in an individual file. The choice of exposure time (0.1 s) guarantees that even the sharpest and fastest moving bands in typical CE runs are not broadened due to blurring. Naturally, it is possible to eventually synchronize the reading of the $C C D$ with the movement of the bands and gain an order of magnitude in sensitivity. 18 The time between exposures $(0.8 \mathrm{~s})$ is dictated by the camera-to-disk transfer rate of our particular computer hardware and software $(0.5 \mathrm{~s})$ and our attempt to limit the total amount of data collected. The total storage space for the 400 data files collected during a run was under 4 megabytes. With proper binning and using state-of-the-art $C C D$ hardware and software, a data rate of $10 \mathrm{~Hz}$ can be achieved. Using a simple BASIC algorithm, the intensities of selected $3 \times 5$ element regions representing each separation capillary were summed. These time dependent sums are then plotted as electropherograms. Separation. Silica capillaries (Polymicro Technologies, Phoenix, AZ, $75 \mu \mathrm{m}$ i.d., $150 \mu \mathrm{m}$ o.d., \#TSP075150, length $=27 \mathrm{~cm}$ ) were prepared by removing $1 \mathrm{~cm}$ 
of polyimide coating with boiling sulfuric acid $25 \mathrm{~cm}$ from the injection end. Equilibration consisted of flushing with $0.1 \mathrm{M} \mathrm{NaOH}$ followed by filling with running buffer (10 $\mathrm{mM}$ bicarbonate adjusted to $\mathrm{pH}=9$ ) and applying a $5 \mathrm{kV}$ potential for at least 10 hours prior to fiber insertion. Solutions of riboflavin (BioRad) and fluorescein (Eastman Kodak) were prepared in running buffer. The electrophoretic separation was driven at $+7.5 \mathrm{kV}$ using a high voltage power supply (Glassman, whitehouse station, NJ, model PS/MJ30P0400-11) with a platinum electrode at the high voltage end and a chrommel electrode at ground. 


\section{RESULTS}

General Considerations. Perhaps the most serious limitation of axial detection schemes concerns photochemical bleaching. This can result from the relatively long exposure of the analyte band to the intense excitation beam. Proper attention to this problem in instrument design is essential. Our earlier experiments, 15 where light was propagated along the entire capillary using total internal reflectance, utilized an organic absorber, orange $G$, to block photochemical damage until the analyte reached tine detection region. In the present case, with partial internal reflection, 18 only a small fraction of the incident laser intensity remains in the capillary after 1 $\mathrm{cm}$ of travel. A small bend (2 cm radius) in the capillary after the detection region prohibits any further light travel thus rendering the use of an organic absorber unnecessary. An additional electronic shutter in the beam path, which opens synchronously with the CCD shutter, blocks the incident beam during the $80 \%$ of time when no signal is being collected and should greatly reduce photochemical damage in the detection region. The lack of photochemical bleaching is confirmed by comparing peak 
heights in single capillaries with different amounts of cumulative exposure to laser excitation.

The relative ease of capillary mounting and optical alignment shown previously has been preserved in this apparatus. To replace a single capillary in the array, two brackets are loosened, the malfunctioning or broken capillary is removed and a new capillary slid into its place. Assuming that the fiber bundle has already been positioned properly relative to the microscope objective, alignment of the excitation beam involves nothing more than the insertion of the optical fiber into the new capillary. Although this can be accomplished by hand even with unaided vision, use of the microscope, which is already in place, speeds up the process. The distance between the tip of the optical fiber and the observation zone does affect the net excitation intensity. This is not critical, however, since variations can be corrected for by calibration standards. Crosstalk between the separation channels is another important design consideration in multiplexed detectors. This must be eliminated or reduced to a predetermined level before applications such as DNA sequencing can be addressed. Crosstalk has two causes: signal light reflections from the walls of adjacent capillaries and scatter from optics inside the microscope. When 
capillaries are placed next to each other, false peaks are observed in nearby capillaries, as shown in Figure 2. Our approach is simply to place spacers, which are the same $150 \mu \mathrm{m} 0 . \mathrm{d}$. capillaries coated with black ink, between each of the separation capillaries. Using this method we reduced crosstalk to well below $1 \%$ of the observed signal. Naturally, more sophisticated masks can be built to eliminate crosstalk completely.

Mobility and Quantitation. Electropherograms for ten parallel separations of riboflavin and fluorescein are shown in Figure 3. The retention times for the two components are similar to those reported previously.19 This indicates that the electroosmotic flow normally present in an open capillary is not significantly disturbed by fiber insertion. The individual retention times in the 10 capillaries vary noticeably. The relative standard deviations (RSD) for the migration times of riboflavin $\left(t_{1}\right)$ and for the migration times of fluorescein $\left(t_{2}\right)$ are around $3 \%$ and $5.5 \%$ respectively. Since the 10 capillaries are unrelated, even the relative migration times of fluorescein $\left(t_{2} / t_{1}\right)$, show a $4 \%$ RSD. Also, the calculated mobilities ( $\mu$ ) of fluorescein (related to $1 / t_{2}-1 / t_{1}$ ) give a RSD of 5\%. Repeat experiments show similar results. This is to be expected given the uncontrolled nature of the surfaces 


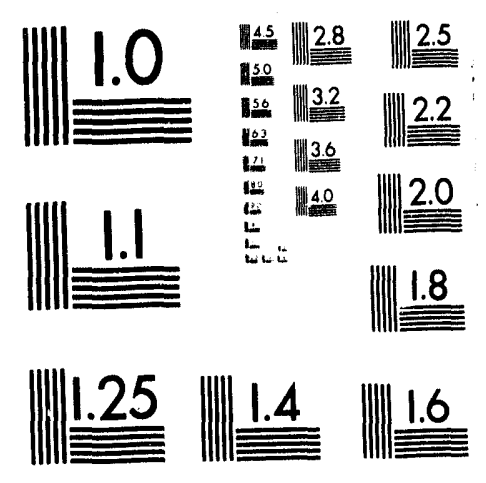



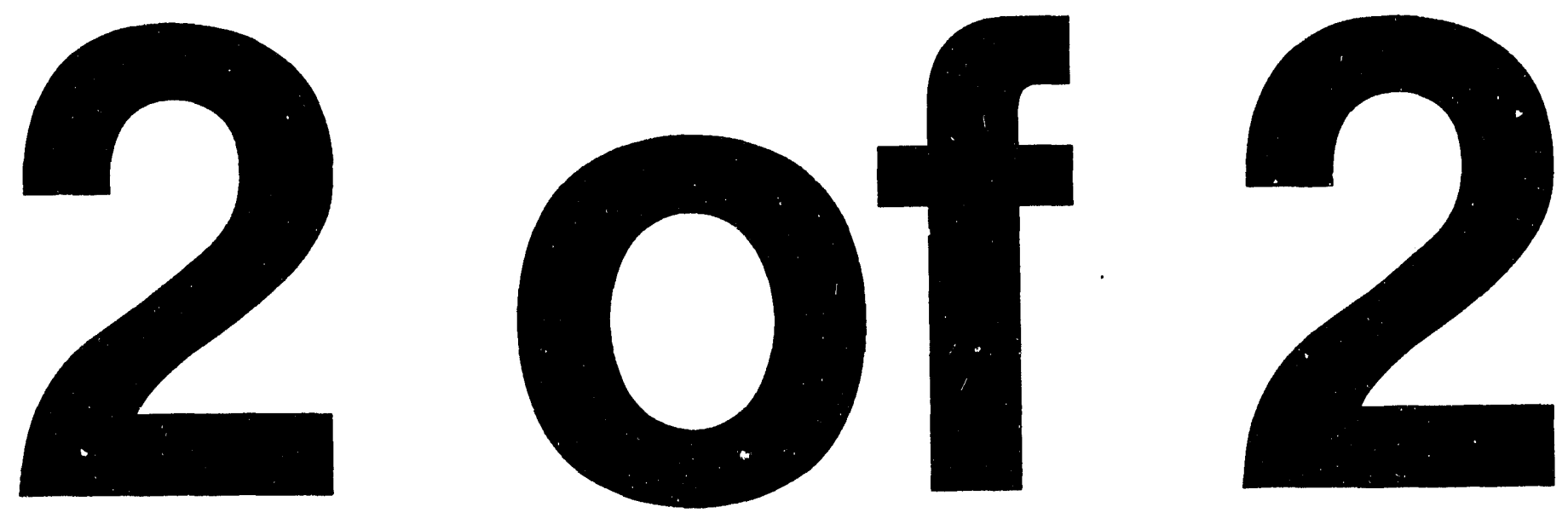


\section{8}

Figure 2. Crosstalk between separation channels in the absence of spacers. Thirteen capillaries were placed side by side and numbered consecutively. Peaks $A$ and $B$ are reflections of the fluorescence signal in capillary 9, C and $D$ arise from the signal in capillary 11 , and.E represents interference from capillary 6. The eluted fluorophore is 3,3'-diethylthiadicarbocyanine iodide (DTDCI), appearing at different times due to variations among the capillaries. The scale is deliberately magnified to show the artifacts. 
Fluorescence Intensity /arbitrary units

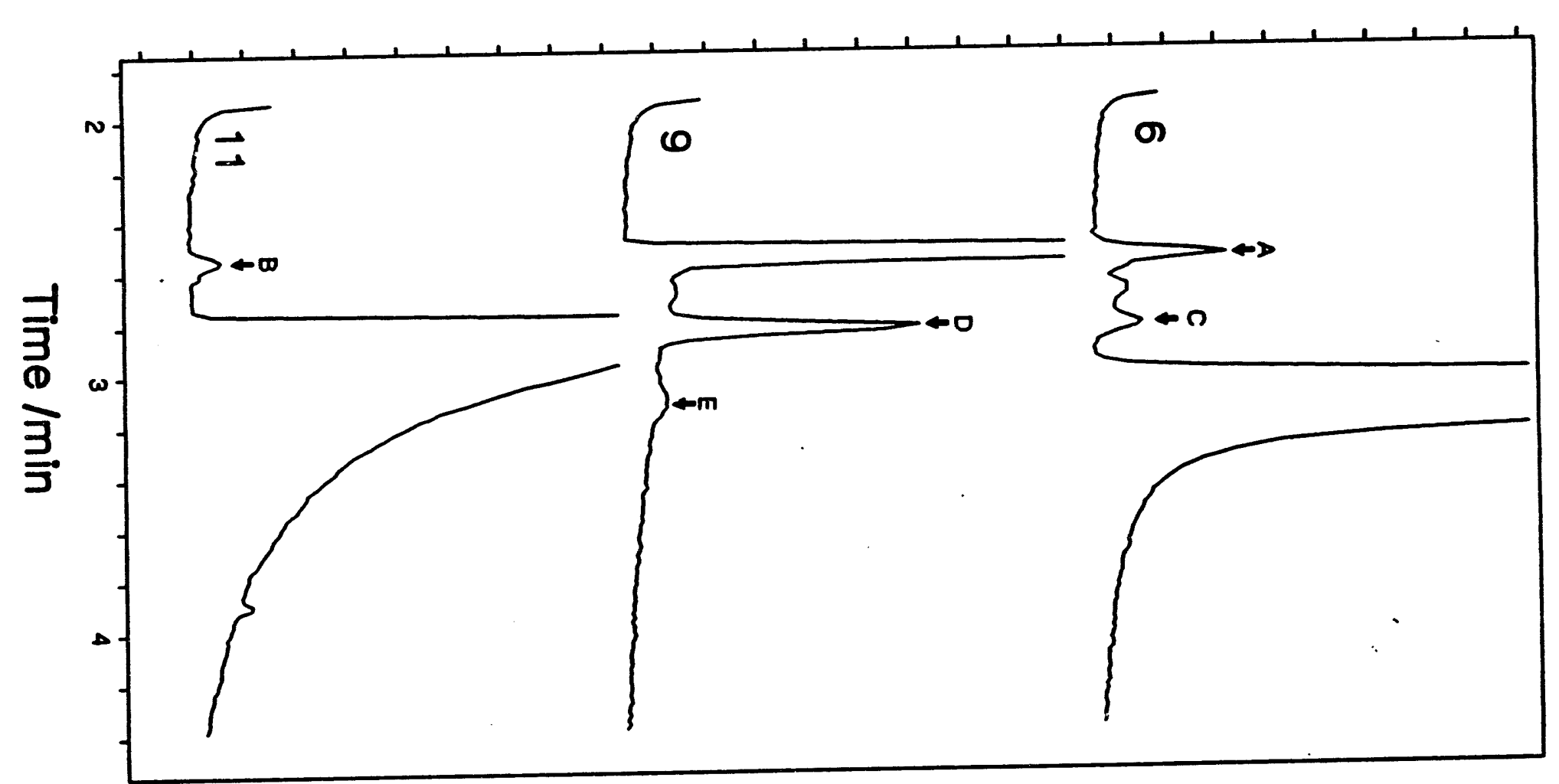


90

Figure 3. Simultaneous electrophoretic separations of riboflavin and fluorescein. Riboflavin concentration, $5 \times 10^{-5} \mathrm{M}$; fluorescein concentration, $6 \times 10^{-7} \mathrm{M}$; injection time a $7500 \mathrm{~V}, 5 \mathrm{~s}$; time axis is in minutes. 

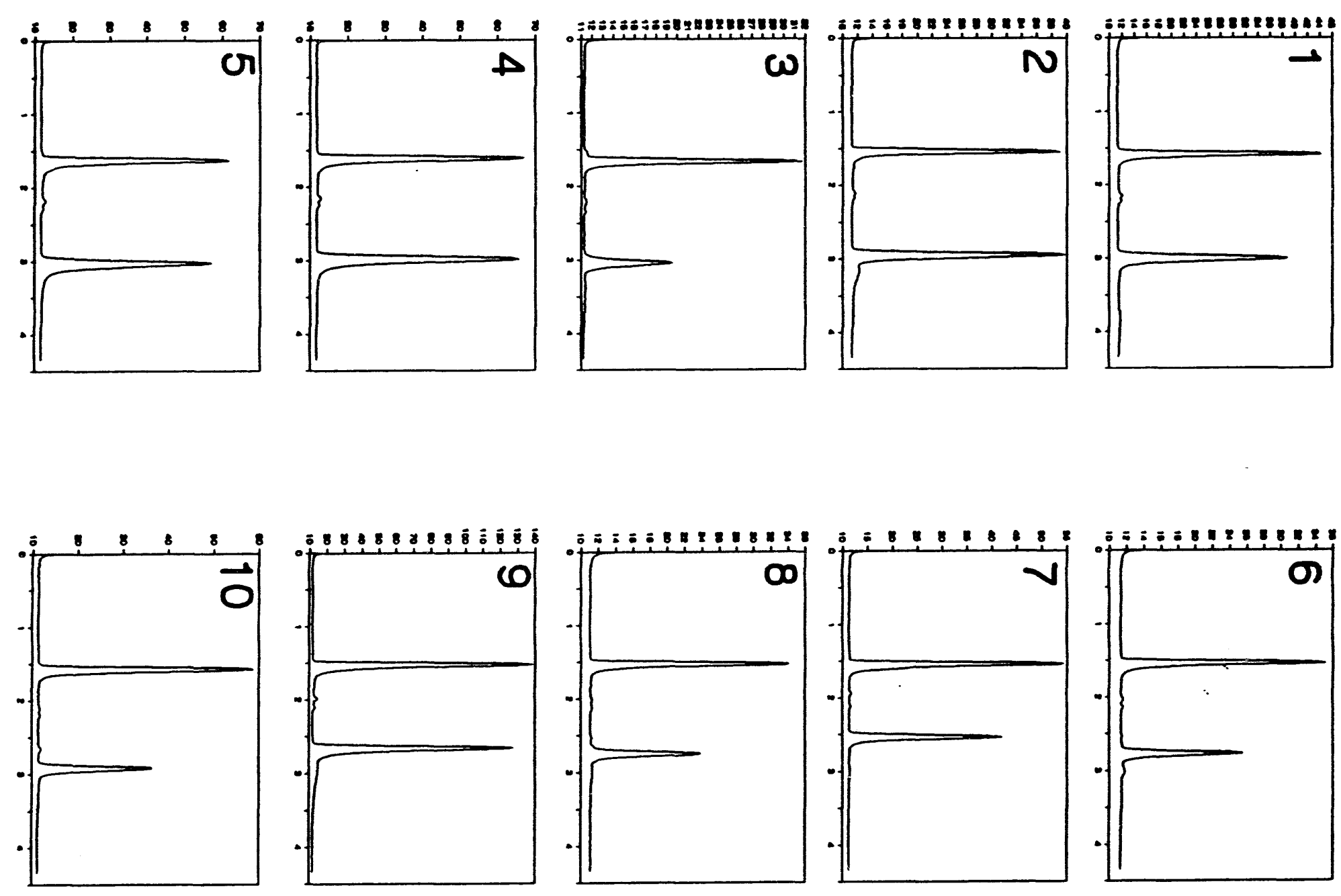
inside each capillary, variations in temperature, variations in capillary i.d., variations in fiber-optic o.d., and differences in the length of insertion of the fiber. However, for a given capillary, run-to-run reproducibility was much better. The ranges of RSD for $t_{1}$, $t_{2}, t_{2} / t_{1}$, and $\mu$ are 0.48 to $1.18,0.48$ to $3.48,0.48$ to 2.48 , and 0.48 to 2.48 respectively. These are not too different from typical run-to-run variations in capillary electrophoresis in the absence of temperature control and for constant voltage operation.

Fig. 3 also shows large variations in the peak areas among the 10 parallel capillaries. The RSD ranges from $70 \%$ for riboflavin $\left(A_{1}\right), 958$ for fluorescein $\left(A_{2}\right)$, and $29 \%$ for the relative areas $\left(A_{2} / A_{1}\right)$. Large differences are expected due to non-uniform coupling of excitation energy into each capillary and variations in pixel sensitivities across the CCD. These dominate over the variations of individual capillary surfaces and geometries. For a given capillary, run-to-run reproducibility (RSD ranges) for $A_{1}, A_{2}$, and $A_{2} / A_{1}$ are 68 to $28 \%, 6 \%$ to 298 , and 3.68 to 178 respectively. Injection bias is responsible for these variations. These are more pronounced than variations in migration times because factors like unequal time constants for electromigration injection, ubiquitous injection, 20 and 
the particular characteristics of the capillary entrance affect the injected amount but not the migration times. It is interesting to note that the peak areas are not correlated with the migration times. This indicates that photochemical bleaching 15 is not important in these experiments. 


\section{DI8CU8sION}

Several important observations can be made. First, truly simultaneous multiplexing of capillary electrophoresis was achieved because the CCD camera looks at all capillaries at all times, with data acquisition rates fast enough for sequencing at $>1$ base per $\mathbf{s}$ per lane. Second, there are no moving parts and the injection (HV) end of the capillary bundle can be freely manipulated without affecting alignment. Third, the $5 \mathrm{~mW}$ excitation laser simply irradiates the entrance of the optical fiber bundle without critical alignment of the optics to achieve distribution of energy into each capillary. Fourth, there are variations in the excitation energies reaching each capillary, but that can be calibrated for in the same way the individual CCD pixels are normalized. Fifth, there are variations in absolute and relative migration times for the two compounds in the set of electropherograms. This is expected due to the uncontrolled nature of the capillary walls (electroosmotic flow), different temperatures, and different geometries in each capillary. However, recently we developed a migration index and an adjusted migration index to specifically correct for these variations. 21 These corrections can be implemented in the next generation 
of the instrument. Sixth, there are variations in the relative peak heights and areas among the capillaries for the same injected sample concentration. This is again expected due to bias at injection due to differences in electroosmotic flow rates, geometries, and injection time constants of the capillaries. Recently,20 we developed a correction scheme to address this exact problem and have been able to reduce injection bias to $<5 \%$, which is adequate for DNA sequencing. Alternatively, an approach based on two internal standards can be used.22 These corrections can be incorporated in future experiments. We expect this scheme to be capable of true multiplexing of 1024 capillaries in DNA sequencing runs. This number is based on the number of column elements already available in modern solid-state imagers. An optical system can be devised based on our results for 10 capillaries. Fiber bundles to 1000 are readily available at a low cost. A 1-5 W argon ion laser can be used to illuminate one end of the fiber bundle, conveniently distributing 0.4 to $2 \mathrm{~mW}$ of light to each fiber. The other end of the fiber bundle can be fanned out into a flat sheet on a guide, which is simply a set of parallel grooves to fix the location of each fiber to maintain a constant spacing. 1024 separation capillaries can also form a flat 
sheet on another guide. Insertion of the fibers (<50 mm) into the capillaries $(75 \mathrm{~mm})$ should then be straightforward. The imaging optics can be a standard distortion-free camera lens, matching each of the optical windows on the capillaries to each pixel column on the detector. Several rows of pixels can be binned 16-18 to provide increased dynamic range without degrading resolution. The data rate of $C C D$ cameras, even in the high-sensitivity, slow-scan mode, can be around $10 \mathrm{~Hz}$. since all channels are monitored at all times, true multiplexing is achieved. At a later stage, one can convert this to a charge-injection device (CID) camera. The advantage is that random access of data logging and even non-destructive reads are possible. One should be able to further increase the data rate by an order of magnitude, making the system compatible with any future increases in the speed of CGE. Data storage is also substantially reduced because the information can be evaluated before binning or reading multiple frames. 23 Also, once a base is identified, the other 3 lanes in the set of 4 need not be read or be subjected to data processing. A second-generation imaging design can also include color filters to accommodate the 4-color sequencing process 7,24 utilizing different parts of the CID detector. 
To compensate for slight variations in migration times from one capillary to the next 10 one can use the migration index we developed.21 All that is needed is separate current measurements in each capillary. If series resistors are connected to each of the capillaries, the resulting voltage can be monitored sequentially by an $A / D$ converter and a desktop computer. Our work here shows that measurements every 0.1 s are sufficient. So, this is a simple task for 1000 capillaries using commercially available serial interface boards. The injection bias can also be corrected in an analogous manner 20 to maintain faithful intensity determinations. Our migration index has not yet been applied to sequencing gels. However, we have obtained preliminary results on a small number of sequencing runs. The results are promising in that variations in length, voltage, and diameter are properly accounted for. Naturally, more work to validate this correction scheme is needed, and will be pursued in the future. 


\section{ACRNOWLEDGMENTE}

Ames Laboratory is operated for the U.S. Department of Energy by Iowa State University under Contract No. W-7405-Eng-82. This work was supported by the Director of Energy Research, Office of Health and Environmental Research. 


\section{REFERENCES}

1. Joint U.S. Department of EnergY and U.S. Department of Health and Human Services Report DOE/ER-0452P, "Understanding Our Genetic Inheritance-The U.S. Human Genome Project: The First Five Years," Washington, DC, April 1990.

2. "National Research Council Report of the Committee on Mapping and sequencing the Human Genome," Board of Basic Riology, Commission on Life Sciences, National Academy Press, Washington, DC, 1988.

3. Stegemann, J.; Schwager, C.; Erfle, H.; Hewitt, N.; Voss, H.; Zimmermann, J.; Ansorge, W. Nucleic Acids Res. 1991, 19, 675-6.

4. Brumley, R. I.; Smith, L. M. Nucleic Acid Res. 1991, 19, 412-6.

5. Cohen, A. S.; Najarian, D. R. ; Paulus, A.: Guttman, A.: Smith, J. A.; Karger, B. L. Proc. Natl. Acad. SCi. U.S.A. 1988, $85,9660-3$.

6. Drossman, H. ; Luckey, J. A.; Kostichka, A. J.; D'Cunha, J.; Smith, L. M. Anal. Chem. 1990, 62, 900-903.

7. Swerdlow, H.; Zhang, J. Z.; Chen, D. Y.; Harke, H. R.; Grey, R.; Wu, S.; Dovichi, N. J.; Fuller, C. 
Anal. Chem. 1991, 63, 2835-2841.

8. Church, G. M.; Kieffer-Higgins, S. Science 1988, 240, $185-188$.

9. Arlinghaus, H. F.: Thonnard, N.; Spaar, M. T.: Sachleben, R. A.; Larimer, F. W.; Foote, R. S.: Woychik, R. P.; Brown, G. M.; Sloop, F. V.: Jacobson, K. B. Anal. Chem. 1991, 63, 402-407.

10. Huang, X. C.; Quesada, M. A.; Mathies, R. A. Anal. Chem. 1992, 64, 967-972.

11. Hjerten, S., Paper M-3, EuChem Conference on Capillary Electroseparations, storlien, Sweden, 1991.

12. Yeung, E. S.; Sepaniak, M. J. Anal. Chem. 1980, 52, $1465 \mathrm{~A}-1481 \mathrm{~A}$.

13. Hernandez, L.: Marquina, R.: Escalona, J.: Guzman, N. J. Chromatogr. 1990, 502, 247-255.

14. Hernandez, L.; Escalona, J.; Joshi, N.; Guzman, N. J. Chromatogr. 1991, 559, 193-196.

15. Taylor, J. A.; Yeung, E. S. Anal. Chem. 1992, 64, 1741-1744.

16. Sweedler, J. V.; Bilhorn, R. B.; Epperson, P. M.; Sims, G. R.; Denton, M. B. Anal. Chem. 1988, 60, $282 A-291 A$.

17. Epperson, P. M.; Sweedler, J. V.; Bilhorn, R. B.: 
Sims, G. R. ; Denton, M. B. Anal. Chem. 1988, 60, $327 A-335 A$.

18. Sweedler, J. V.; Shear, J. B.; Fishman, H. A.: Zare, R. N.; Scheller, R. H. Anal. Chem. 1991, 63, 496-502.

19. Whang, C. W.: Yeung, E. S. Anal. Chem. 1992, 64, $502-506$.

20. Lee, T. T.; Yeung, E. S. Anal. Chem. 1992, 64, 1226-1231.

21. Lee, T. T.; Yeung, E. S. Anal. Chem. 1991, 63, 2842-2848.

22. Dose, E. V. ; Guichon, G. A. Anal. Chem. 1991, 63, 1154-1158.

23. Koutny, L. B.; Yeung, E. S., An Expert System for Data Acquisition to Achieve Constant Signal-to-Noise: Application to Imaging of DNA Sequencing Gels, Anal. Chem. (AC920455+), submitted.

24. Smith, L. M.: Sanders, J. Z.; Kaiser, R. J.; Hughes, P.; Dodd, C.; Connell, C. R.; Heiner, C.; Kent, S. B. ; Hood, L. E. Nature 1986, 321, 674-679. 
PAPER 4 INVESTIGATION OF ELECTROKINETIC FLOW USING CHARGE-COUPLED DEVICE IMAGING 
INVESTIGATION OF ELECTROKINETIC FLOW USING CHARGE-COUPLED DEVICE IMAGING

John A. Taylor and Edward S. Yeung*

Ames Laboratory-USDOE and Department of Chemistry Iowa State University, Ames, IA 50011 


\section{ABSTRACT}

An imaging system based on microscope optics and a charge coupled device phototransducer is used to form high resolution images of fused silica capillaries. This technique provides an unmistakable empirical examination of the fluid motion inside the capillary under electrokinetic and pressure induced (Poiseuille) conditions. Two experiments are described. The first is observation of a front of fluorescent labeled solvent as it travels through the capillary. The second is an examination of the flow profile using submicron-sized particles as probes. 


\section{INTRODUCTION}

Electroosmotic flow is a phenomenon that arises from a the formation of a double layer of complimentary charges at a surface. One set of charges is fixed in place and the other is mobile, free to move with the attraction or repulsion of an applied electrical field. Early interest among analytical chemists was in harnessing electroosmosis to drive flow through chromatographic packings.5,6 This approach offered greatly reduced band broadening without backpressure. The explosive development of capillary electrophoresis and electrokinetic chromatography has caused much discussion and theoretical speculation about electroosmosis. Most recently, several researchers have reported approaches to controlling this flow. These methods range from variation of electrolyte equilibria and temperature ${ }^{1}$ to direct application of an electric field to the capillary surface. 2,3

One of the most heavily cited early references offering a theoretical perspective on these phenomena was published by Rice and whitehead in 1965.4 The relation between linear velocity and radial position was derived as: 


$$
v(r)=-\Omega E \quad 1-\frac{I(k r)}{I(k a)}
$$

where: $v(r)=1$ inear velocity

$$
\begin{aligned}
\Omega= & \text { constant for a particular liquid and solid } \\
& \text { phase } \\
\mathbf{E}= & \text { applied electrical field } \\
\mathbf{I}= & \text { zero-order modified Bessel function of the } \\
& \text { first kind proportional to the potential at a } \\
& \text { specified location along the capillary radius } \\
\mathbf{k}= & \text { reciprocal of the double-layer thickness } \\
\mathbf{a}= & \text { capillary radius }
\end{aligned}
$$

If $\mathrm{ka}$ is large, which is the case for a capillary internal diameter of $75 \mu \mathrm{m}$ and an electrical double layer of less than $10 \mathrm{~nm}, 8$ the I term becomes insignificant. This predicts a flat flow profile for fluid in the capillary in all but the small double layer region.

Imaging through a micxoscope onto a charge-coupled device ( $C C D)$, has been used before to image flow in the junction between two capillaries. 7 obtaining a radial profile of the flow velocity inside the capillary, however, requires special consideration of lensing caused by curvature of the capillary walls. The work to be described also utilizes fluorescence measurements rather than absorbance measurements. 
EXPERIMENTAL

The microscope-based CCD imaging system with fiber optic beam introduction (fig. 1) was similar to that reported previously. In this case, however, a single capillary was placed between a microscope slide and supported cover glass and surrounded with fluid. This refractive index modifying mount was essential to obtaining high magnification in images as well as a narrow depth of field and greatly reduced scatter from capillary walls. Another difference is the microscope (AO Spencer) which was used to obtain a higher magnification than the former Stereo Zoom model. A more powerful laser (Uniphase) was also useful in increasing the particle fluorescence signal well over the dark current noise limit.

\section{Elow generation}

The scale of events being recorded dictated large differences in flow velocities between the two experiments. Band fronts ranged from 1-3 mm in length while the measurable movement of a single particle was limited to the $200 \mu \mathrm{m}$ that could be imaged in one frame.

Hydrostatic flow was produced by elevating one end of the capillary $50 \mathrm{~cm}$ for the fluorescent band studies and $0.7 \mathrm{~cm}$ for the particle migration measurements. Electrokinetic flow was driven with a high voltage power 
Figure 1.

Schematic for CCD imaging of fused silica capillaries. CS, glass cover slip; CAP, fused silica capillary: OF, optical fiber: GS, glass microscope slide; SP, spacers made from glass cover slips; CCD, charge coupled devise camera; 5X, magnifying camera adapter; 10X, 20X, microscope objectives: MB, microscope body: GND, grounded buffer reservoir; HV, buffer reservoir held at high voltage or elevated for hydrostatic flow. 


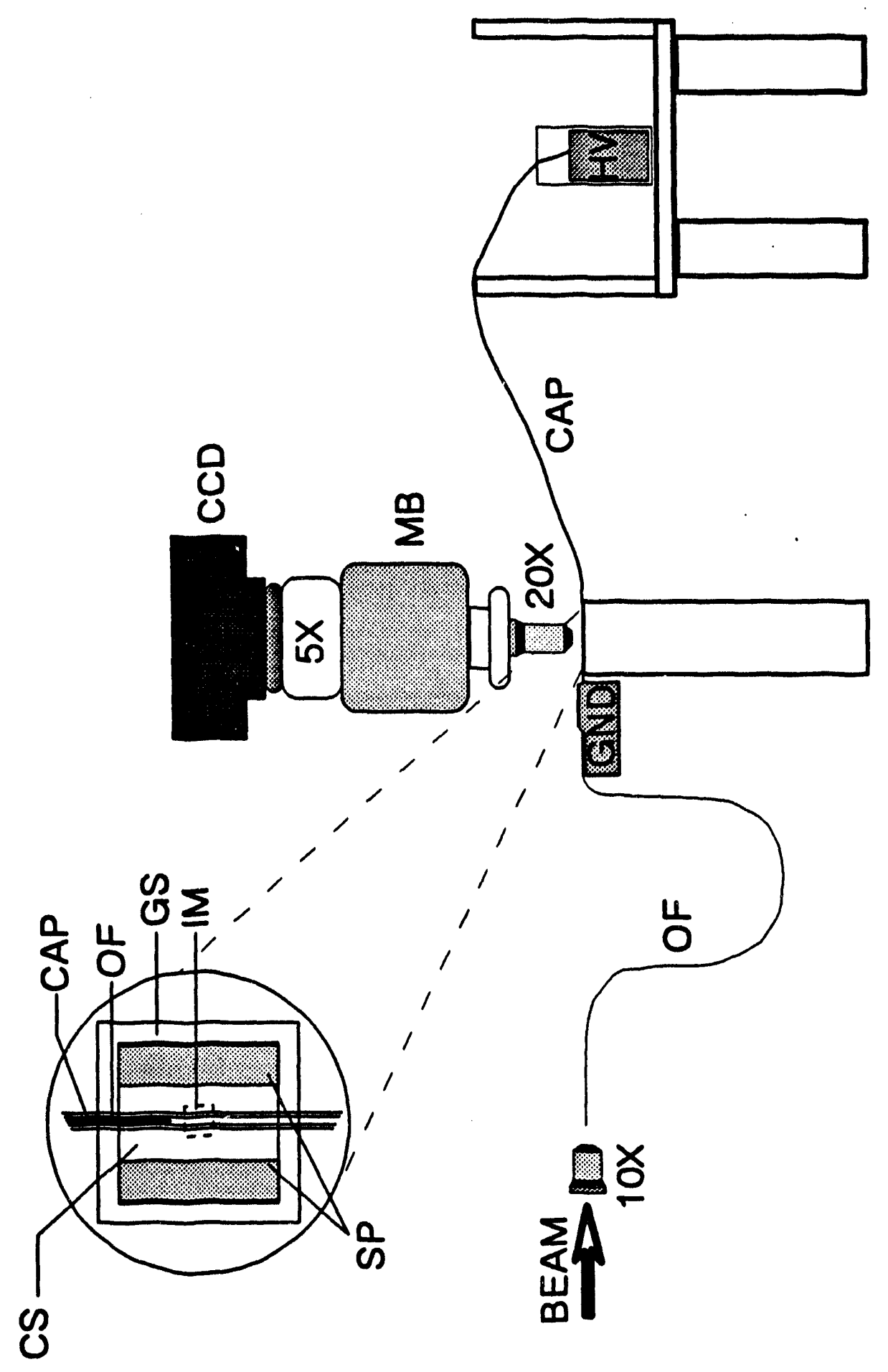


supply (Glassman) set at $10 \mathrm{kV}$ for band measurements and $+120 \mathrm{~V}$ (minimum output) for the particle studies at $\mathrm{pH}=7$ and $-120 \mathrm{~V}$ at $\mathrm{pH}=3$.

Image acouisition and analysis

A different strategy for data acquisition and analysis was applied to each experiment. For the first experiment, ten rows $(5 \mu \mathrm{m})$ of the capillary image were binned along the capillary axis and recorded every $1.2 \mathrm{~s}$ during the travel of the fluorescent band through the detection region. In the particle experiments, unbinned images of a $100 \mu \mathrm{m}$ X $200 \mu \mathrm{m}$ region were collected at 5 second intervals with an exposure time of 1.5 seconds. The resulting particle fluorescence streaks in a 15-20 $\mu \mathrm{m}$ focal plane could be easily differentiated from out-of-focus particles or other anomalies and were measured manually from the CCD system high-resolution monitor.

\section{Solutions and buffers}

All buffer solutions were $10 \mathrm{mM}$ bicarbonate. These solutions were adjusted to the indicated $\mathrm{pH}$ with $\mathrm{HCl}$, degassed under vacuum in an ultrasonic bath, and filtered through $0.22 \mu \mathrm{m}$ syringe filters. Carboxylate-modified fluorescing latex microspheres $(282 \mathrm{~nm}$ diameter) were obtained from Interfacial Dynamics and diluted 1000 to 5000 fold in the bicarbonate buffer. 


\section{RESULT8 AND DI8COS8ION}

The most important factor in planning these experiments was the necessity of depth resolution perpendicular to the capillary axis. In studying the flow inside a cylindrical capillary, only a thin, twodimensional plane including the capillary axis will yield accurate information about the radial dependence of flow velocity. In this case, a common light microscope was used with $100 x$ magnification to obtain a depth of field of around $20 \mathrm{~mm}$. Spectral information from outside this thin focal plane was either lost into the background or easily discriminated from in-focus information by appearance.

Three-dimensional plots can be drawn (figure 2) which show the fraction of peak fluorescence intensity reached in the image as a function of radial position and time during the emergence of a fluorescent front. In order to compensate for variations in pixel response and illumination, each pixel of each frame was normalized to the last frame of the sequence. The capillary walls appear as a series of mountains and valleys because the peak intensities in these regions remained close to the dark current of the sensor.

A few important observations are apparent in these plots. First, even though the rates of travel for the two 
I

112

Figure 2. Three dimensional plots of normalized fluorescence intensity. Top, gravity flow, $\mathrm{pH}=7$; bottom, electrokinetic flow, $\mathrm{pH}=7$. 

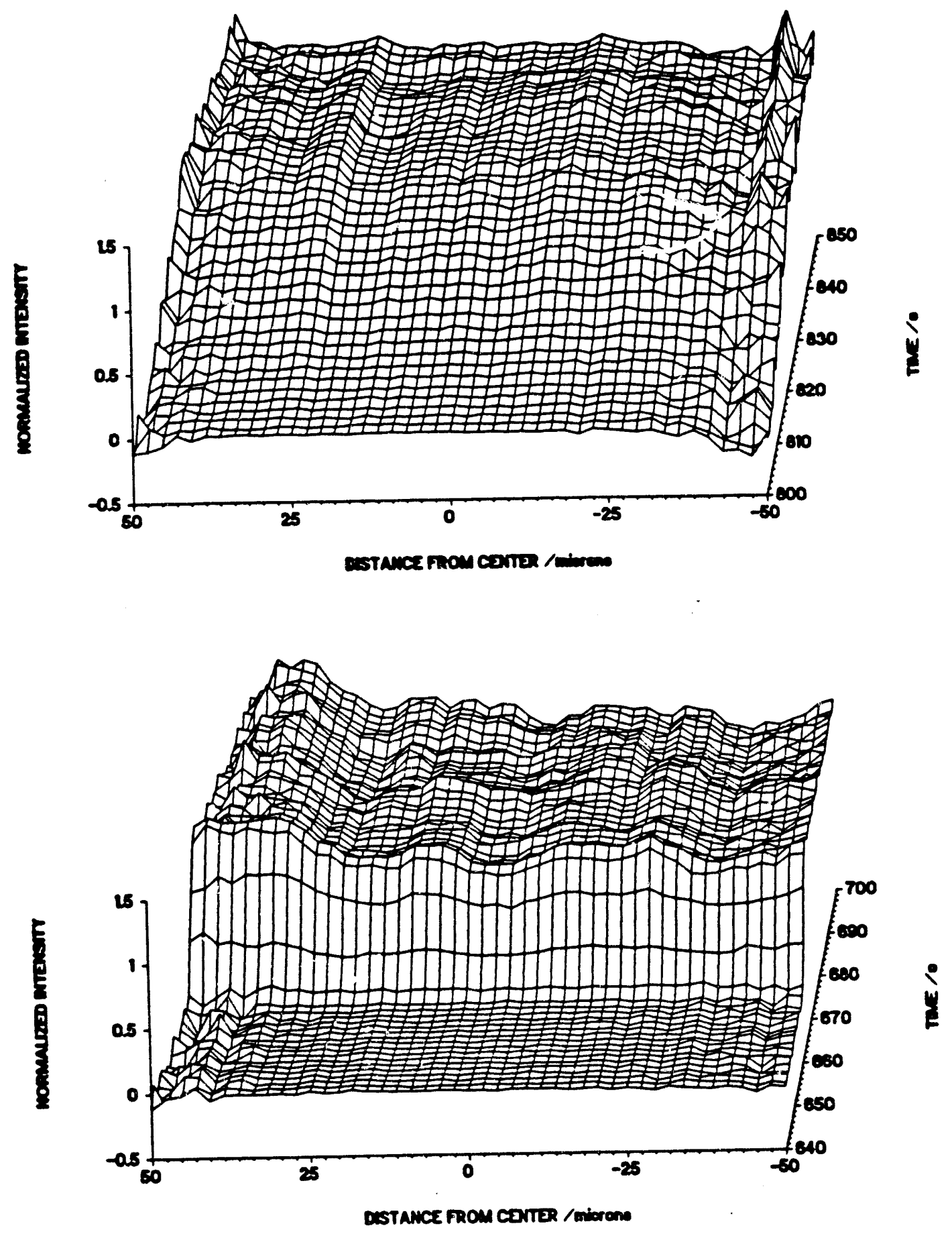
band fronts are roughly the same (within 10\%), the rise time for the Poiseuille front is almost a factor of two longer than that for the electrokinetic front. This is consistent with observations of increased band broadening caused by axial dispersion when pressure driven flow is operative. second, the normalized intensity as the front emerges is constant across the capillary radius for both flow regimes. This supports the idea tha radial diffusion is fast enough to "average" the riboflavin concentration across the capillary.

While the relatively fast diffusion of molecular fluorophores prevents measurement of linear velocity as a function of radius, sub-micron sized particles, which do not diffuse quickly, can be used to trace the flow. The time-lapsed images collected (figure 3) show streaks which define the travel of the particle through the capillary during the time of exposure. streak length is proportional to particle velocity in along the $x$-axis. Pointers a-e indicate streaks that were measured to determine the velocity profile in cases of electrokinetic and gravity generated flow. The streaks in each figure have different widths $(\mathrm{c}>\mathrm{a}>\mathrm{b}$; $\mathrm{d}>\mathrm{e}$ ). This may indicate additional motion in the $y$-direction and within the imaging plane. This difference in width appears to be related to the length and location of the streak along the 
115

Figure 3. Images of particle generated streaks. 1, gravity flow $\mathrm{pH}=7 ; 2$, eletrokinetic flow, $\mathrm{pH}=7$. a-e, measurable streaks; 0 , out-offocus streaks. 


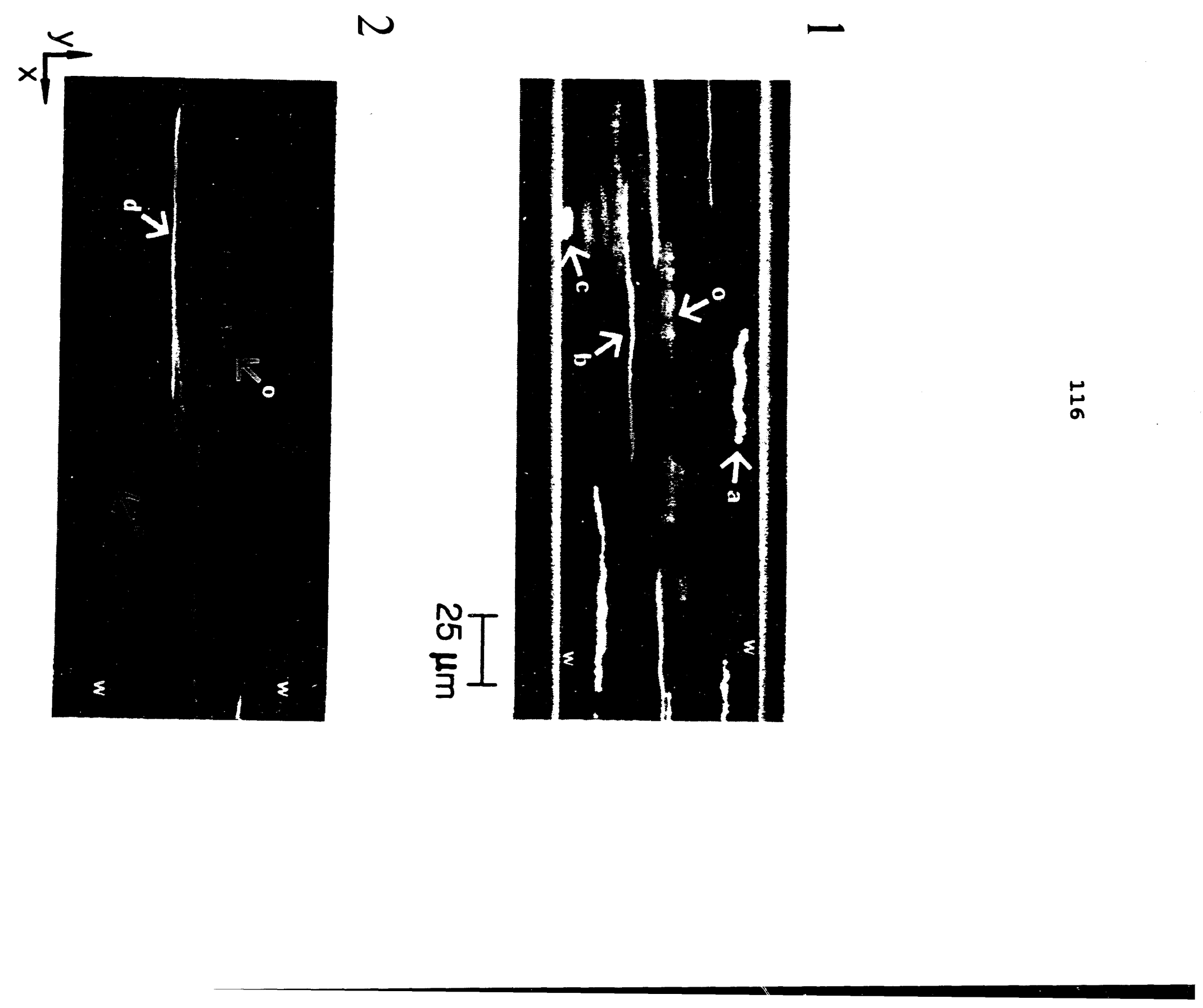


capillary axis and should not be confused with particles which are out-of-focus (indicated by the 0 pointers).

As figure 4 indicates, in the pressure-driven mode (44a) particle velocity approaches zero near the capillary walls. This is the effect predicted by Poiseuille's law. When electroosmotic flow is operative $(4 b, c)$, however, particle velocity remains constant across the width of the capillary which approximates the electroosmotic profile described earlier.

of interest is the apparent dip present in fig. $4 \mathrm{~b}$ which represents the distribution at $\mathrm{pH}=7$. This effect is more pronounced at higher $\mathrm{pH}$ (larger zeta potential) and indicates complicating factors. One explanation is that the liquid phase viscosity causes the bulk solution in the center of the capillary to drag behind the mobile portion of the double layer near the walls. Another is that the mobility of the particles is affected in some way by thermal gradients inside the capillary. The extent of this effect may be dictated ionic strength and the applied potential field. Further experimentation will be necessary to determine which description is operative. 
118

Figure 4. Scatter plots of normalized streak length vs. radial distance from capillary center in $\mu \mathrm{m}$. $a$, gravity flow $\mathrm{pH}=7 ; \mathrm{b}$, electrokinetic flow, $\mathrm{pH}=7 ; \mathrm{c}$, electorkinetic flow, $\mathrm{pH}=3$. 

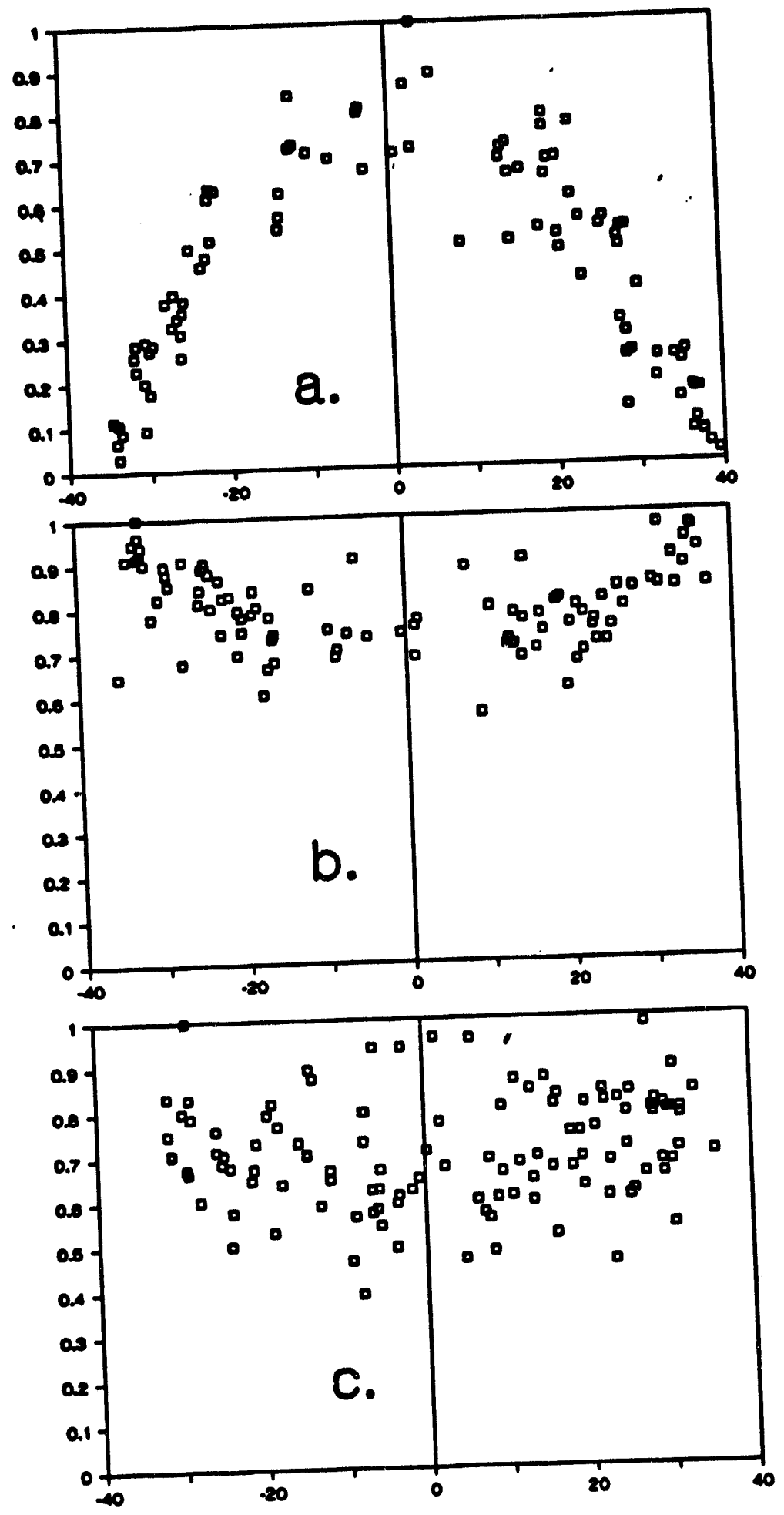


\section{IITERATURE CITED}

1. Chang, H.-T.; Yeung, E.S. Anal, Chem. 1993, 65, $650-652$.

2. Wu. C.-T.; Huang, T.-L.; Lee, C.S.; Miller, C.J. Anal. Chem. 1993, 65, 568-571.

3. Hayes, M.A.; Kheterpal, I.; Ewing, A.G. Anal. Chem. $1993,65,27-31$.

4. Rice, C.L.; Whitehead, R. The Journal of Physical Chemistry 1965, 69(11), 4017-4024.

5. Pretorius, V.; Hopkins, B.J.; Schieke, J.D. Journal of Chromatography 1974, 23-30.

6. Stevens, T.S.; Cortes, H.J. Anal. Chem. 1983, 55, $1365-1370$.

7. Kuhr, W.G.; Licklider, L.; Amankwa, L. Anal. Chem. $2993,65,227-282$.

8. Ewing, A.G.; Wallingford, R.A.; Olefirowicz, T.M. Anal. Chem. 1989, 61, 292A-303A. 


\section{GENERAL CONCLUBIONB}

The papers of this thesis have demonstrated that onaxis beam introduction is a viable approach to meeting many of the challenges of CE detection. In addition, the directions taken in this research have shed light on areas of interest such as electrophoresis in organic solvents, interactions between capillary separations run in parallel, and the nature of electroosmotic flow.

Axial absorbance detection showed a 15 fold enhancement of absorbance signals by using the entire length of the sample band as analytical pathlength. This principle was later applied to commercial instruments using ultraviolet wavelengths and more traditional light sources by $x$ iaobing $x i$ of the Yeung group. 21

Axial fluorescence detection and its application to the human genome project brought about the first truly multiplexed CE detection system. A patent is now in process for the instrument described. Kyoji Ueno is currently using the microscope-based CCD imaging system with cylindrical lenses and other optics to optimize beam introduction for multiple capillary detection.22 The immediate goal is to demonstrate 100 simultaneous separations in a manner that can be scaled up by a factor of ten. 


\section{LITERATURE CITED}

1. Walbroeh1, Y.; Jorgenson, J.W. I. Chromatogr. 1984, $315,135-143$.

2. Foret, F.; Demi, M.; Vladislav, K.; Bocek, P. Electrophoresis $1986,7,430-432$.

3. Wang, T.; Hartwick, R.A.; Champlin, P.B. I. Chromatogr. $1989,462,147-154$.

4. Xi, X.; Yeung, E.S. Anal. Chem. 1990, 62, 15801585.

5. Kuhr, W.G. Anal. Chem. 1990, 62, 403R-414R.

6. Dovichi, N.J.; Martin, J.C.; Jett, J.H.; Keller, R.A. Science $1983,845-847$.

7. U.S. Department of Energy and U.S. Department of Health and Human Services Understanding our Genetic Inheritance, The U.S. Human Genome Project: The First Five Years Report DOE/ER-0452P, April, 1990.

8. Bishop, J.E.; Waldholz, M. Genome; Simon and Schuster: New York, 1990.

9. Kevles, D.J. In The Code of Codes; Daniel J. Kevles; Leroy Hood, Ed.; Harvard University Press: Cambridge, $1992 ;$ pp.3-36.

10. National Research Council Mapping and sequencing the Human Genome; National Academy Press: Washington, 1988 . 
11. Davis, J. Mapping the code; John wiley \& Sons: New York, 1990.

12. Smith, L. M.; Sanders, J.Z.; Kaiser, R.J.; Huges, P.: Dodd, C. : Connell, C.R.; Helner, C.; Kent, S.B.; Hood, I.E. Nature $1986,321,674-679$.

13. Swerdlow, H.: Zhang, J.Z.; Chen, D.Y.: Harke, H.R.: Grey, R.: Wu, S.: Dovichi, N.J.: Fuller, C. Anale Chem. 1991, 63, 2835-2841.

14. Cohen, A.S.; Najarian, D.R.: Paulus, A.; Guttman, A.: Smith, J.A.: Karger, B.L. Proc. Natl. Acad. Sci. U.S.A. 1988,85 9660-3.

15. Church, G.M.: Kieffer-Higgins, S. Science 2988, $240,185-188$.

16. Xue, Y.: Yeung, E.S. submitted to Analytical Chemistry.

17. Hobbs, P.C.D. Optics and Photonics 1991, 17-23.

18. Hogan, B.L.; Yeung, E.S. Trends in Analytical Chemistry 1993, 12, 4-9.

19. Hogan, B.L.; Yeung, E.S. Analytical Chemistry 1992, $64,2841-2845$.

20. McGregor, D.A.: Yeung, E.S. submitted to I. Chromatogr.

21. Xi, X.; Yeung, E.S. Applied Spectroscopy 1991, 45, 1199-1203.

22. Ueno, K., personal communication. 


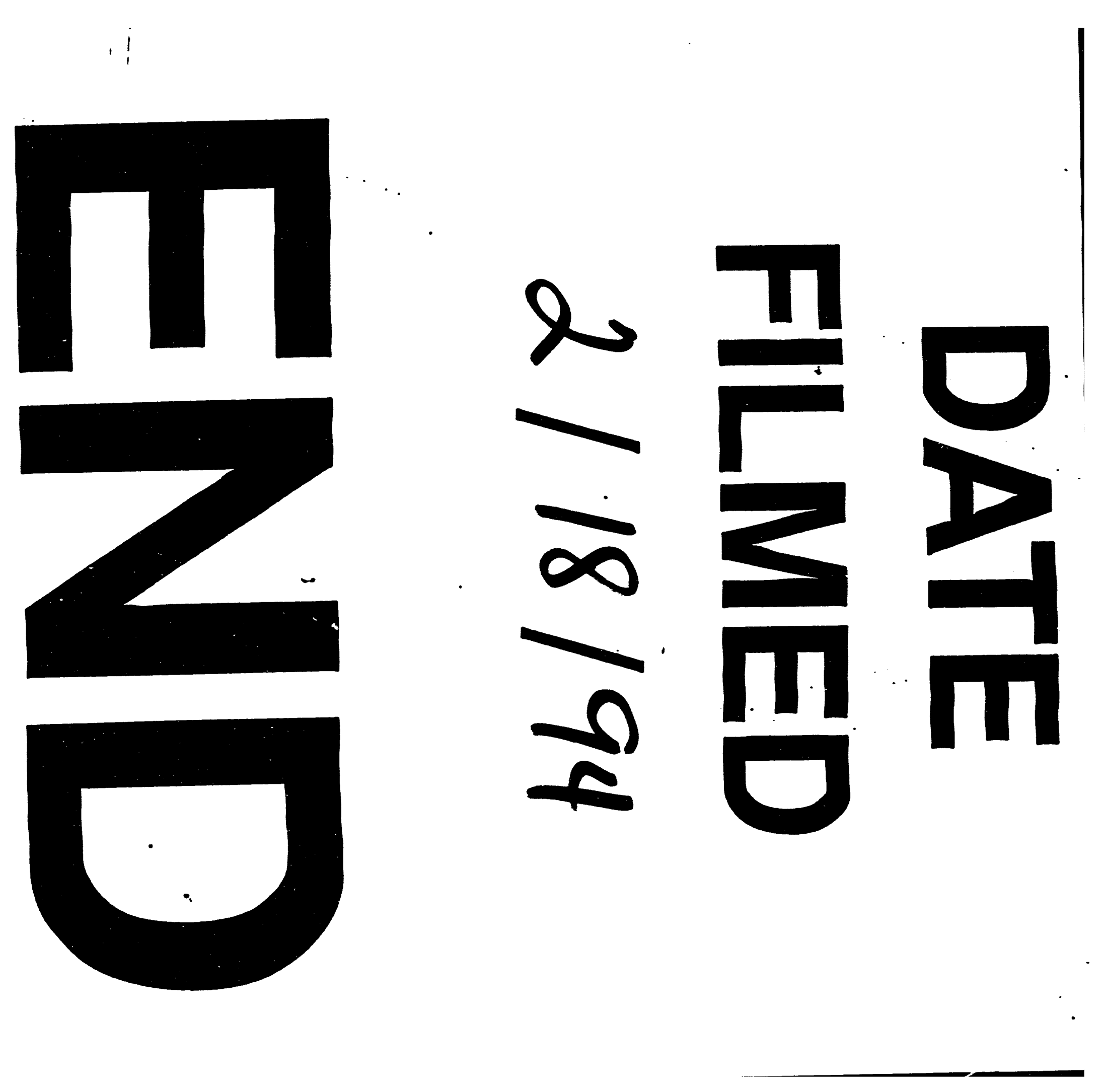


